\title{
Discovery of SARS-CoV-2 antiviral drugs through large-scale compound repurposing
}

https://doi.org/10.1038/s41586-020-2577-1

Received: 20 April 2020

Accepted: 17 July 2020

Published online: 24 July 2020

Check for updates

\author{
Laura Riva ${ }^{1,23}$, Shuofeng Yuan ${ }^{2,3,4,23}$, Xin Yin', Laura Martin-Sancho', Naoko Matsunaga', \\ Lars Pache', Sebastian Burgstaller-Muehlbacher ${ }^{5}$, Paul D. De Jesus', Peter Teriete', \\ Mitchell V. Hull ${ }^{6}$, Max W. Chang ${ }^{7}$, Jasper Fuk-Woo Chan ${ }^{2,3,4}$, Jianli Cao ${ }^{2,3,4}$, \\ Vincent Kwok-Man Poon ${ }^{2,3,4}$, Kristina M. Herbert', Kuoyuan Cheng ${ }^{8,9}$, Tu-Trinh H. Nguyen ${ }^{6}$, \\ Andrey Rubanov', Yuan Pu', Courtney Nguyen', Angela Choi ${ }^{10,11,12}$, Raveen Rathnasinghe ${ }^{10,11,12}$, \\ Michael Schotsaert ${ }^{10,11}$, Lisa Miorin ${ }^{10,11}$, Marion Dejosez ${ }^{13}$, Thomas P. Zwaka ${ }^{13}$, Ko-Yung Sit ${ }^{14}$, \\ Luis Martinez-Sobrido ${ }^{15}$, Wen-Chun Liu ${ }^{10,11}$, Kris M. White ${ }^{10,11}$, Mackenzie E. Chapman ${ }^{16}$, \\ Emma K. Lendy ${ }^{17}$, Richard J. Glynne ${ }^{18}$, Randy Albrecht ${ }^{10,11}$, Eytan Ruppin ${ }^{8}$, \\ Andrew D. Mesecar ${ }^{16,17}$, Jeffrey R. Johnson ${ }^{10}$, Christopher Benner ${ }^{7}$, Ren Sun ${ }^{19}$, \\ Peter G. Schultz ${ }^{6}$, Andrew I. Su ${ }^{20}$, Adolfo García-Sastre ${ }^{10,11,21,22}$, Arnab K. Chatterjee $^{6 凶}$, \\ Kwok-Yung Yuen ${ }^{2,3,4} \bowtie$ \& Sumit K. Chanda ${ }^{1 凶}$
}

\begin{abstract}
The emergence of severe acute respiratory syndrome coronavirus 2 (SARS-CoV-2) in 2019 has triggered an ongoing global pandemic of the severe pneumonia-like disease coronavirus disease 2019 (COVID-19) ${ }^{1}$. The development of a vaccine is likely to take at least 12-18 months, and the typical timeline for approval of a new antiviral therapeutic agent can exceed 10 years. Thus, repurposing of known drugs could substantially accelerate the deployment of new therapies for COVID-19. Here we profiled a library of drugs encompassing approximately 12,000 clinical-stage or Food and Drug Administration (FDA)-approved small molecules to identify candidate therapeutic drugs for COVID-19. We report the identification of 100 molecules that inhibit viral replication of SARS-CoV-2, including 21 drugs that exhibit dose-response relationships. Of these, thirteen were found to harbour effective concentrations commensurate with probable achievable therapeutic doses in patients, including the PIKfyve kinase inhibitor apilimod ${ }^{2-4}$ and the cysteine protease inhibitors MDL-28170, Z LVG CHN2, VBY-825 and ONO 5334. Notably, MDL-28170, ONO 5334 and apilimod were found to antagonize viral replication in human pneumocyte-like cells derived from induced pluripotent stem cells, and apilimod also demonstrated antiviral efficacy in a primary human lung explant model. Since most of the molecules identified in this study have already advanced into the clinic, their known pharmacological and human safety profiles will enable accelerated preclinical and clinical evaluation of these drugs for the treatment of COVID-19.
\end{abstract}

In January 2020, SARS-CoV-2 was identified as the causative agent of the COVID-19 outbreak first detected in Wuhan, China ${ }^{1}$.SARS-CoV-2 is an enveloped, positive-sense, single-stranded RNA Betacoronavirus, related to the viruses that caused the SARS outbreaks in 2002 to 2004 and Middle East respiratory syndrome (MERS) outbreaks since 2012. The World Health Organization (WHO) declared COVID-19 a pandemic on 11 March 2020, and as of 3 September 2020, more than 25.88 million confirmed cases and 859,000 deaths have been recorded in 216 countries 5 .

Production of a vaccine for SARS-CoV-2 is anticipated to take 12-18 months ${ }^{6}$, and de novo development of antiviral therapies usually requires $10-17$ years $^{7}$. Therefore, repositioning of clinically evaluated drugs represents one of the most promising strategies for the rapid identification and deployment of treatments for emerging infectious diseases such as COVID-19. Towards this end, clinical investigations have focused on the repurposing of several approved antiviral therapies, including the HIV-1 protease inhibitors lopinavir and ritonavir (https:// clinicaltrials.gov), the hepatitis $\mathrm{C}$ virus protease inhibitor danoprevir ${ }^{8}$ and the influenza antiviral favipiravir (https://clinicaltrials.gov). Additionally, remdesivir, a viral RNA polymerase inhibitor ${ }^{9}$, has been granted emergency use authorization by the FDA for the treatment of COVID-19 on the basis of clinical trial data demonstrating a reduction in time to recovery ${ }^{10,11}$.

While these targeted repurposing strategies provide potentially rapid trajectories towards an approved treatment, additional therapies for SARS-CoV-2 infection are required to enhance clinical efficacy, expand worldwide drug supplies, and address the potential emergence of viral resistance. An unbiased large-scale evaluation of known drugs may identify additional unanticipated therapeutic options that can be positioned for accelerated preclinical and clinical evaluation. Here we 
describe a high-throughput reprofiling screen using the ReFRAME (repurposing, focused rescue and accelerated medchem) drug library, a comprehensive open-access library of around 12,000 drugs that have been either FDA-approved, registered outside the USA, entered clinical trials or undergone substantial preclinical characterization ${ }^{12}$, to identify existing drugs that harbour antiviral activity against SARS-CoV-2 in a cell-based assay ${ }^{12,13}$. The ReFRAME library has previously been used to successfully identify potential therapies for tuberculosis ${ }^{14}$, Cryptosporidium $^{15}$ and fibrosis ${ }^{16}$. Each of the compounds in this collection has been previously optimized for efficacy, safety and bioavailability. This means that considerable investments have already been made in research and development of these compounds, which will shorten the drug discovery and development timeline ${ }^{17}$. Using this approach, we identified 100 drugs that inhibit SARS-CoV-2 replication in mammalian cells, including 21 compounds for which a dose-response relationship with antiviral activity could be established. Rapid experimental and clinical evaluation of these therapeutic compounds for in vivo antiviral efficacy and amelioration of disease-associated pathologies may provide an important opportunity for the accelerated development of potential therapies for COVID-19.

\section{Development of a high-throughput screen}

Given the urgent need for therapeutic agents to treat SARS-CoV-2 infection, we developed a high-throughput assay to enable large-scale screening of known drugs. Vero E6 cells are kidney epithelial cells from an African green monkey that have been shown to be highly permissive to SARS-CoV-2 infection ${ }^{18}$ and viral replication in these cells can be assessed through measurement of viral-induced cytopathogenic effects $^{19}$ (CPE). A clinical isolate of the SARS-CoV-2 virus (SARS-CoV-2 HKU-001a) ${ }^{20}$ was used for assay development and screening. Assay parameters, including cell seeding density, multiplicity of infection (MOI) and time points, were optimized in Vero E6 cells by measuring virus-induced CPE in a 384-well format.

To assess robustness and reproducibility of the optimized assay in a high-throughput screening (HTS) configuration, we initially evaluated the assay using the collection of known bioactive molecules LOPAC1280. To our knowledge, no compound with activity against SARS-CoV-2 in Vero E6 cells had been reported at the time this effort was initiated. On the basis of studies indicating that inhibition of the PIKfyve kinase inhibits entry of viruses such as Ebola $^{21,22}$, we evaluated and confirmed the potential antiviral activity of the PIKfyve kinase inhibitor APY0201 against SARS-CoV-2 (Extended Data Fig. 1a). This enabled us to benchmark the dynamic range of the assay on the basis of a reliable positive control. SARS-CoV-2-induced CPE activities corresponding to each well were normalized to the median of each plate $\left(\log _{2}\right.$ (fold change)). The average $Z^{\prime}$ factor for the replicate screens was 0.4 , and the correlation coefficient $\left(R^{2}\right)$ was 0.81 (Extended Data Fig. 1b, c). Twenty-eight compounds were selected for further confirmation on the basis of activities in replicate screens (Extended Data Fig. 1b, red circles). These included the HIV protease inhibitor nelfinavir mesylate hydrate and the antagonist of the serotonin receptors 5-HT1B and 5-HT1D, GR 127935 hydrochloride hydrate, which has been shown to efficiently block infection by SARS-CoV and SARS-CoV- $2^{23-27}$.

\section{HTS analysis of a known-drug library}

Having established that these assay conditions were suitable for progression towards a large-scale screen, we used this experimental design to screen the comprehensive ReFRAME drug-repurposing collection (Fig. 1a). Specifically, we assessed the potential antiviral activity of 11,987 compounds against SARS-CoV-2 in Vero E6 cells. The assay, conducted at a final compound concentration of $5 \mu \mathrm{M}$ was designed to capture multicycle replication, based on low viral input $(\mathrm{MOI}=0.01)$ and an extended end-point measurement ( $72 \mathrm{~h}$ after infection). We a

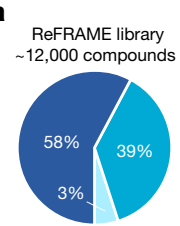

SARS-CoV-2 infection
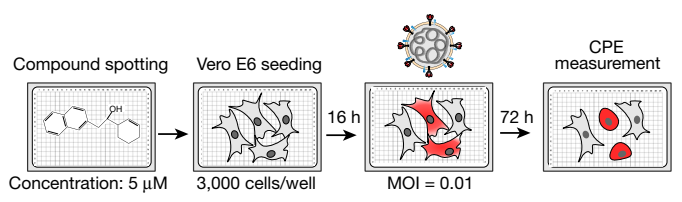

Investigational new drugs

Pre-clinicalconpous

b Controls

c
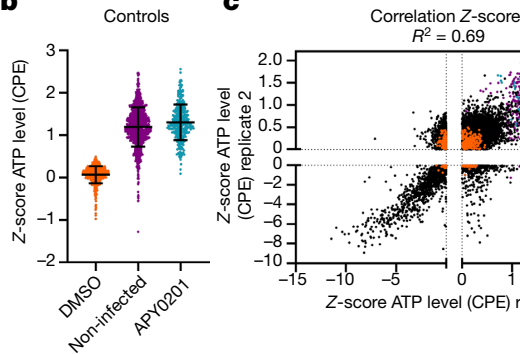

$R^{2}=0.69$

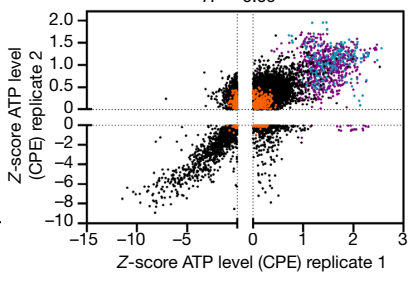

d

Distribution Z-score

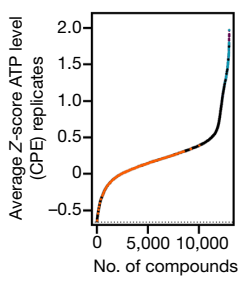

Fig. 1 | High-throughput ReFRAME collection drug repositioning screen for SARS-CoV-2 antiviral compounds. a, A schematic of the screening strategy used for the repositioning analysis of the ReFRAME library. Classification of the approximately 12,000 compounds in the ReFRAME collection across different stages of clinical development is depicted in the pie chart. For the HTS, compounds were pre-spotted in 384-well plates at a final concentration of $5 \mu \mathrm{M}$. Next, 3,000 Vero E6 cells were added to each well and pre-incubated with each compound for $16 \mathrm{~h}$, followed by infection with a clinical isolate of SARS-CoV-2 (HKU-001a) with a MOI of 0.01. ATP levels in each well were measured $72 \mathrm{~h}$ after infection using a Cell Titer Glo viability assay as a surrogate measurement of viral CPE. $\mathbf{b}, Z$-scores after normalization to the median of each plate for all positive (APY0201) and negative (DMSO) controls, as well as for non-infected cells, across all the screening plates. Data are mean \pm s.d. for at least 376 independent wells. c, Correlation plot indicates the activity ( $Z$-score) of each compound in the two replicate screens. d, The activity distribution of each compound based on the average of the $Z$-score of each replicate. Each dot indicates the average $Z$-score of the screening replicates for each drug (black dots). Values corresponding to DMSO (orange dots), APY0201 (cyan dots) and non-infected cells (purple dots) are also represented. $R^{2}$ indicates the linear correlation coefficients for the replicates (c).

observed a reasonable dynamic range between positive and negative controls (Fig. 1b, d, Extended Data Fig. 1d, f) and a positive correlation between replicates $\left(R^{2}=0.69\right)$ (Fig. 1c, Extended Data Fig. 1e), enabling the identification of compounds with potential antiviral activities (see Supplementary Discussion).

We next evaluated enrichment of known targets and target classes among the screen data. Using a gene set enrichment analysis (GSEA) tool, we examined the distribution of antiviral activities of the compounds within individual target classes to determine whether certain therapeutic mechanisms returned more active compounds than would be expected by chance ${ }^{28,29}$. We found 15 target classes and 51 drug targets that were enriched in the ranked hit list (Fig. 2a, Extended Data Figs. 2, 3, Supplementary Table 1), including modulators of benzodiazepine receptors, aldose reductase, potassium channels, cholesterol homeostasis, serine proteases and retinoic acid receptor agonists. Of note, we observed that inhibition of viral replication by retinoic acid receptor agonist tazarotene could be reversed through direct chemical antagonism of the transcriptional activator retinoic acid receptor (RAR) antagonist Ro41-5253 (Fig. 2b). Additionally, RNA-sequencing (RNA-seq) analysis performed on Vero E6 cells to assess the transcriptional effects of viral challenge in this system revealed a significant decrease $(P=0.0006)$ in the mRNA levels of genes related to retinol metabolism after infection with SARS-CoV-2 (Supplementary Table2). These data are consistent with previously reported RNA-seq analysis of nasopharyngeal swabs ${ }^{30}$ (Supplementary Table 2), and suggest that 


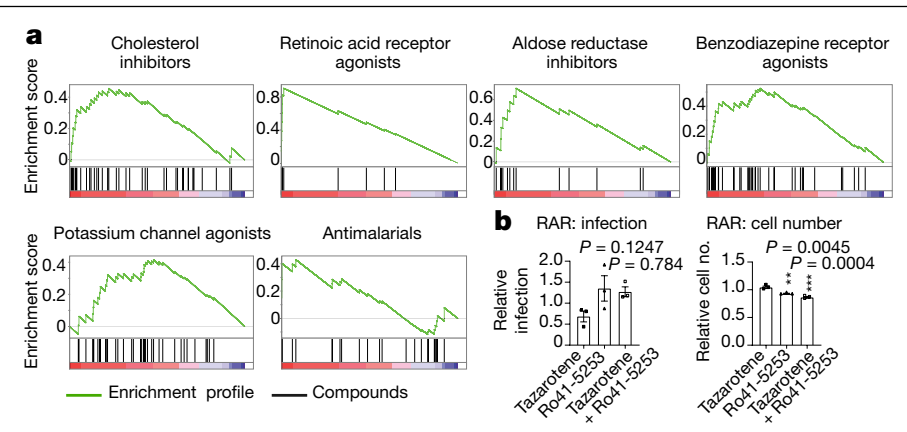

Fig. 2 | GSEA and target gene expression. a, Enriched targets and mechanisms of action of potential antiviral compounds were determined through GSEA. GSEA enrichment plots provide the distribution of the enrichment score (green line) across compounds that were annotated to molecular targets, ranked in order of antiviral activities (left to right). Vertical black lines reflect the position of each compound within a specific target class across the ranked dataset, where the leftmost position indicates most potent antiviral activity (red), and the rightmost position indicates inactivity in the HTS screen (blue). Enriched target clusters are shown, including retinoic acid receptor agonists, benzodiazepine receptor inhibitors, aldose reductase agonists, potassium channel agonists, cholesterol inhibitors and antimalarials $(P<0.05$, false discovery rate (FDR) $q<0.33$ ). Additional enriched target classes are shown in Extended Data Fig. 2.Pvalues were calculated as indicated in the Methods. b, Chemical epistasis analysis of retinoic acid receptor agonist antiviral activity. Left, Vero E6 cells were treated with $5 \mu \mathrm{M}$ of the RAR agonist tazarotene and challenged with SARS-CoV-2, and infection was determined as described in Fig. 3. Similarly, Vero E6 cells where pretreated with $5 \mu \mathrm{M}$ of the RAR antagonist Ro41-5253, either alone or in combination with $5 \mu \mathrm{M}$ of tazarotene (left). Cellular toxicity was measured by counting cell numbers (right). Data are normalized to the mean of DMSO-treated wells and represent mean \pm s.e.m. for $n=3$ independent experiments. One-way ANOVA followed by Dunnett post-test. ${ }^{* *} P \leq 0.01$ and ${ }^{* * *} P \leq 0.001$.

retinol metabolism and signalling may act as a critical host-pathogen interaction circuit in controlling viral infection.

To elucidate the expression pattern of the molecular targets of putative antiviral compounds from this cell-based screen, we used a previously reported dataset to analyse the transcriptional profile of these genes across cell types within the respiratory tract $^{31}$. Critically, a majority of the mapped targets of active compounds were also expressed in relevant respiratory epithelial cells, suggesting that these may be physiologically relevant drug targets (Extended Data Figs. 4, 5).

\section{Orthogonal validation of selected compounds}

Approximately 300 compounds were identified for validation studies on the basis of criteria outlined in the Methods and Supplementary Discussion. We assessed the activity of selected hits at 2.5 and $1 \mu \mathrm{M}$, in contrast to the $5 \mu \mathrm{M}$ concentrations used in the original screen, using an orthogonal assay readout. Specifically, Vero E6 cells treated with selected compounds were challenged with a different SARS-CoV-2 isolate (SARS-CoV-2 USA-WA1/2020), and viral infection was directly quantified by immunostaining for the virally encoded nucleoprotein (NP). This configuration probably biased this validation towards confirmation of early inhibitors (Supplementary Discussion). Approximately $30 \%$ of the selected compounds ( 100 compounds) were found to reduce viral replication by at least $40 \%$ (Supplementary Table 3 ).

Several validated compounds were members of enriched GSEA target classes (Fig. 2a, Extended Data Fig. 2), including retinoic acid receptor agonists (LGD-1550, tretinoin, tamibarotene, acitretin, tazarotene, AGN-190521, AGN-191659, AM 580, arotinoid acid (TTNPB), EC-23 (AGN190205), MDI-101 and MDI 403), the aldose reductase inhibitor AL 3152, benzodiazepine receptor agonists (NS-11394 (AN-721), saripidem, tetrazepam, ZK-93426, zaleplon GR and pagoclone) and antimalarial drugs (AQ-13, DDD498, ISQ-1, $N$-tert-butylisoquine and hanfangchin A; Supplementary Table 3). In addition, the molecules confirmed to inhibit SARS-CoV-2 replication included six drugs with regulatory approval in the USA or Japan. These include the antimalarial drug chloroquine, the anti-psoriatic molecule acitretin and the anti-histamine astemizole (Supplementary Table 3).

\section{Dose-response and synergy analysis}

Although highly dependent on the pharmacokinetic properties of a compound, therapeutic dose ranges are typically expected to track to cellular half maximal effective concentration $\left(\mathrm{EC}_{50}\right)$ values below $1 \mu \mathrm{M}$. Therefore, we conducted a dose-response analysis to determine the relationship between compound concentration and antiviral activity (Fig. 3a, b, Extended Data Fig. 6b, c). In addition to remdesivir, 20 compounds harboured discernable dose-dependent antiviral activities, most of which could be segregated on the basis of broad functional, structural or target-based classes (Fig. 3a, b, Extended Data Fig. 6b, c). We observed a significant divergence between cellular toxicity and antiviral activities (Fig. 3a, Extended Data Fig. 6a), underscoring that the effect of these compounds on replication is well outside a range where their cytotoxic or cytostatic activities may be influencing measurements of viral growth (Supplementary Table 4).

We next evaluated potential synergies between known drugs confirmed in dose-response studies and remdesivir. Four compounds were observed to have notable levels of synergy with remdesivir, including hanfangchin A (also known as tetrandrine), which was one of the antimalarial compounds validated in this study (Fig. 3c). Hanfangchin $\mathrm{A}$ is a bis-benzylisoquinoline alkaloid that has been shown to inhibit multiple $\mathrm{Ca}^{2+}$ channels ${ }^{32}$, and has previously been reported to antagonize entry of Ebola virus in vitro and in vivo through the targeting of endosomal two-pore channels ${ }^{33}$. However, the cellular antiviral activities of chloroquine derivatives have not been recapitulated in clinical trials ${ }^{34,35}$, possibly owing to the inability to achieve the safe and efficacious concentration required for antiviral activities in patients ${ }^{36}$. These data suggest that a combinatorial approach should be further investigated to determine whether in vivo and clinical synergies exist between the two drugs within acceptable safety margins.

\section{Validation across human cell lines}

We next sought to ensure that observed efficacies were not restricted to Vero E6 cells. We thus evaluated compound efficacies on two additional human cell lines that support SARS-CoV-2 replication. Specifically, we used Huh-7 and HEK293T cells transduced with angiotensin-converting enzyme 2 (ACE2). Dose-titration analysis found that nearly all (19 out of 21) of the evaluated compounds inhibited viral replication in one or both of these cell lines at potencies equivalent to or greater than those observed in Vero E6 cells (Fig. 4a, b, Extended Data Fig. 7a, b). Thirteen compounds exhibited an $\mathrm{EC}_{50}$ value of less than $500 \mathrm{nM}$ in at least one cell line (Fig. 4a, b, Extended Data Fig. 7), suggesting that they inhibit viral replication at doses can may be achievable in vivo. These include the peroxisome proliferator-activated receptor- $\gamma$ (PPAR- $\gamma$ ) agonist DS-6930, which, similar to the RAR agonists, probably regulates a nuclear hormone receptor-dependent transcriptional program that functions to obstruct viral replication. In addition, clinical-stage non-nucleoside HIV-1 reverse transcriptase inhibitor R 82913 inhibited SARS-CoV-2 replication with an $\mathrm{EC}_{50}$ of $210 \mathrm{nM}$, consistent with reported cell-based antiviral activities against HIV-1 ${ }^{37,38}$. Further investigation will be required to determine whether R 82913 inhibits SARS-CoV-2 replication by inhibition of polymerase function or through other mechanisms. Furthermore, clofazimine, an FDA-approved molecule on the WHO Model List of Essential Medicines, showed an antiviral EC ${ }_{50}$ of $310 \mathrm{nM}$. This molecule has anti-mycobacterial and anti-inflammatory activity $^{39}$, and is used for the treatment of leprosy. Further studies are 

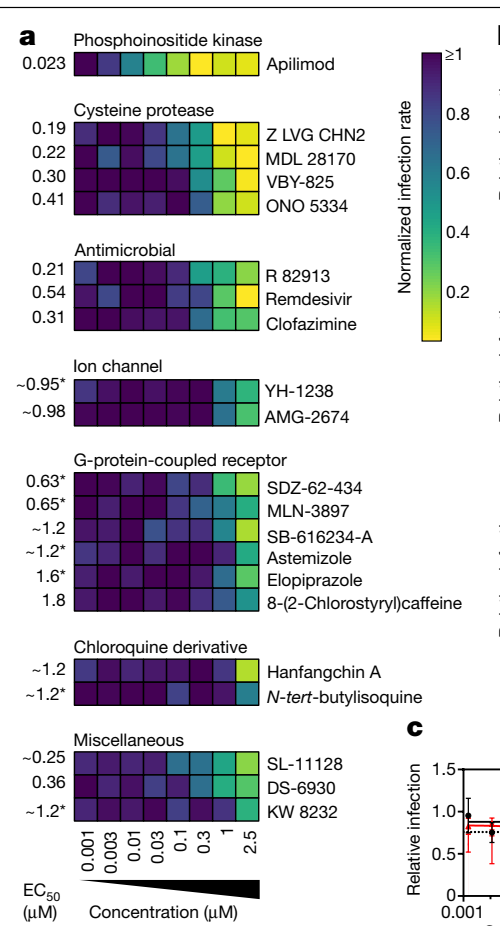
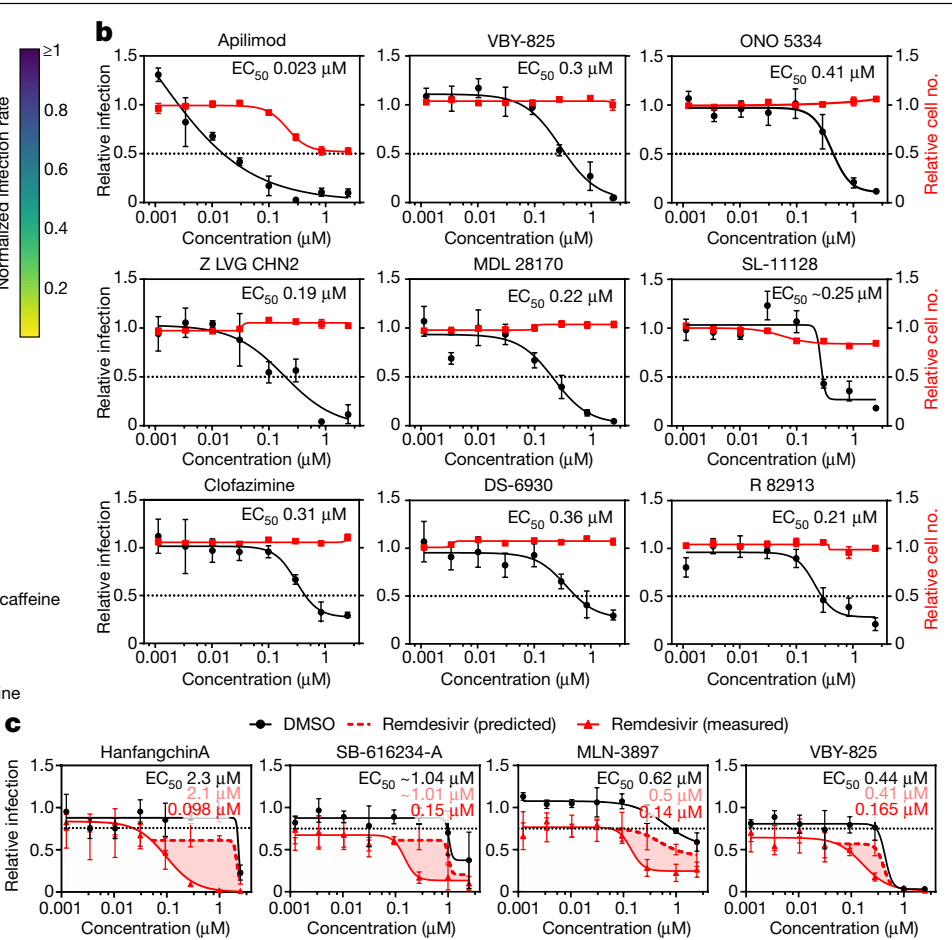

Fig. 3 | Dose-response relationships of selected antiviral compounds and synergy with remdesivir. a-c, Vero E6 cells were pre-treated for $16 \mathrm{~h}$ with increasing concentrations of the indicated compound and then infected with SARS-CoV-2 at a MOI of 0.1. Twenty-four hours after infection, cells were fixed and analysed by immunofluorescence imaging. For each condition, the percentage of infection was calculated as the ratio of the number of infected cells stained for coronavirus NP to number of cells stained with DAPI. a, Heat map representing normalized infection of the indicated 21 compounds in a dose-response analysis, on a scale from 0 to 1 , depicting the average of $n=5$ independent experiments. Compounds are grouped in predicted functional clusters. For MDL 28170 and 8-(2-chlorostyryl)caffeine, $0.85 \mu \mathrm{M}$ was used instead of $1 \mu \mathrm{M}$ at the second highest dose. Extrapolated $\mathrm{EC}_{50}$ values are listed on the left of the heat map. Asterisks indicate compounds for which $\mathrm{EC}_{50}$ values were calculated on the basis of observed values at the highest concentrations. b, Dose-response analysis of the most potent compounds in a, showing

required to understand the mechanism by which this drug blocks the replication of SARS-CoV-2.

\section{Effect of antivirals on SARS-COV-2 life cycle}

We next performed studies to evaluate whether five of the most potent compounds identified in this study, apilimod, VBY-825, ONO 5334, ZLVGCHN2 and MDL 28170, act on entry or post-entry steps of the viral life cycle. We first conducted time-of-addition studies, which compared the effects of the compound administered concurrently with or immediately after viral infection with those of the compound administered two or five hours after viral challenge, which allows time for viral entry (Fig. 5a). To further corroborate these results, we also evaluated the effects of these molecules on infectivity of vesicular stomatitis virus (VSV)-based virus-like particles pseudotyped with SAR-CoV-2 spike (S) protein, MERS S protein or VSV G protein (Fig. 5b). Results from these experiments indicate that these compounds inhibit the entry step of viral replication. The protease inhibitors VBY-825, ONO 5334, Z LVG CHN2 and MDL 28170 were found to lack potent inhibitory activity on SARS-CoV-2 3C-like protease (3CLpro) and papain-like protease (PLpro), indicating that observed antiviral activities are the result of inhibition of host proteases (Extended Data Fig. 8). Z LVG CHN2 targets cysteine proteinases produced by group A streptococci, and has also been shown to suppress herpes simplex virus (HSV) replication by

infectivity (black), cell number (red) and cellular $\mathrm{EC}_{50}$ values (see also Extended Data Fig. 6).c, Compounds at indicated doses were combined with $800 \mathrm{nM}$ remdesivir or a negative control (DMSO), and antiviral dose-response relationships were determined in Vero E6 cells using the experimental conditions described in $\mathbf{b}$. Remdesivir alone inhibited viral infection by $20 \%$ (black dotted line). The predicted additive combinatorial activity of remdesvir and the indicated compound (see Methods) is denoted by a red dotted line. Observed activity of remdesivir in combination with the indicated compound is shown with a solid red line, and shaded portions of graph indicate the difference between predicted and observed combinatorial activities. $\mathrm{EC}_{50}$ for compound alone (black), and predicted (pink) and observed (red) $\mathrm{EC}_{50}$ for the combined treatment are presented. Data are normalized to mean values for DMSO-treated wells and represent mean \pm s.e.m. of $n=3$ (apilimod, MDL 28170, Z LVG CHN2, VBY-825 and SL-11128) (b, c) or $n=5$ (ONO 5334, clofazimine, DS6930 and R82913) (b) independent experiments.

inhibiting theenzymaticactivity ofHSV-encoded cysteineprotease ${ }^{40}$.Thus, Z LVG CHN2 probably acts by inhibition of an endosomal protease, although its precise cellular target is unknown. MDL 28170 is a cathepsin B inhibitor that also impairs infection by SARS-CoV and Ebola virus ${ }^{27,41}$, ONO5334 is a cathepsin Kinhibitor, and VBY-825 acts as a reversible cathepsin protease inhibitor. Human cysteinyl cathepsins are required for the proteolytic processing of virally encoded proteins during infection ${ }^{42-44}$, and cathepsin activity is probably required for proper processing of the SARS-CoV-2 S protein through the endosome to activate its fusogenic acitivity $^{43}$. Of note, ONO 5334 was found to be well tolerated in phase II clinical trials for the treatment of osteoporosis, and its development was discontinued only because of an unfavourable competitive landscape ${ }^{45,46}$.

\section{Evaluation in primary human cell models}

ONO 5334, MDL 28170 and apilimod were further evaluated for antiviral activity in human induced pluripotent stem cell (iPSC)-derived pneumocyte-like cells (see Methods). Cells were differentiated, incubated with the respective compounds, and then challenged with SARS-CoV-2. Treatment with antivirals resulted in significantly decreased viral replication in these primary cell types. ONO 5334, MDL 28170 and apilimod reduced the number of infected cells by $72 \%, 65 \%$ and $85 \%$, respectively (Fig. 4c-e, Extended Data Fig. 9a-c). Finally, we assessed the antiviral activity of apilimod in an ex vivo lung culture 
a 293T-ACE2 cells

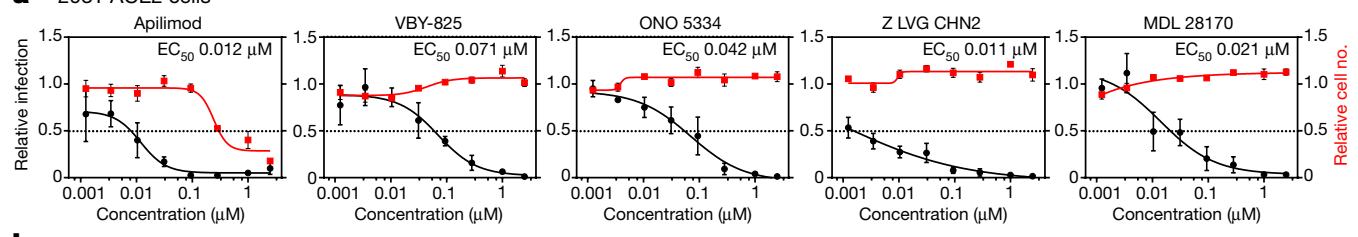

b Huh-7-ACE2 cells
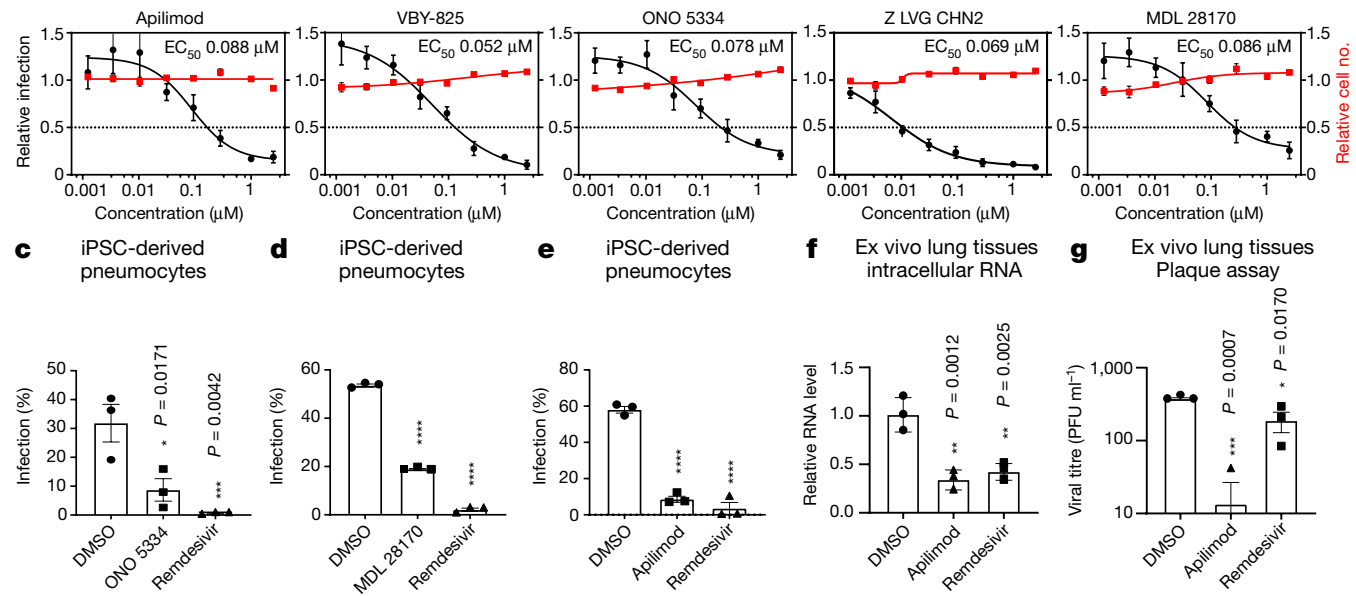

Fig. 4 | Assessment of antiviral activity in human cell models. a, b, HEK293T (a) and Huh-7 cells (b) transduced with ACE2 were pre-treated for $16 \mathrm{~h}$ with increasing concentrations of the indicated compound and then infected with SARS-CoV-2 $(\mathrm{MOI}=0.3(\mathbf{a})$ and $0.2(\mathbf{b}))$. Twenty-four hours after infection, cells were fixed and immunostained, and imaged by immunofluorescence microscopy. For each condition, the percentage of infection was calculated as the ratio of the number of infected cells stained for coronavirus NP to the number of cells stained with DAPI. Compound concentrations ranged between $1 \mathrm{nM}$ and $2.5 \mu \mathrm{M}$. Dose-response curves for infectivity (black) and cell number (red) are shown. Data are normalized to the mean for DMSO-treated wells and represent mean \pm s.e.m. of $n=4$ independent experiments. $\mathrm{EC}_{50}$ for each compound was calculated using a four-parameter logistic nonlinear regression model and is indicated. c-e, iPSC-derived pneumocytes were incubated with $5 \mu \mathrm{M}$ of the indicated compound $2 \mathrm{~h}$ before infection, and then infected with $10^{5}$ plaque-forming units (PFU) of SARS-CoV-2. Two days after infection, cells were collected and viral infection was quantified by staining for coronavirus NP and flow cytometry. Data are mean \pm s.e.m. of $n=3$ biological replicates. One-way ANOVA followed by Dunnett's post-test. $\mathbf{f}, \mathbf{g}$, Ex vivo lung tissues were infected with $5 \times 10^{5}$ PFUSARS-CoV-2. After $2 \mathrm{~h}$, the inoculum was removed and $5 \mu \mathrm{M}$ of the indicated compound was added. Twenty-four hours after infection, supernatants were collected for quantification of viral titre by plaque assay (f) and cells were collected for quantification of intracellular viral RNA (g). Data are mean \pm s.e.m. of $n=3$ biological replicates. One-way ANOVA followed by Dunnett's post-test. ${ }^{*} P \leq 0.05,{ }^{* *} P \leq 0.01,{ }^{* * *} P \leq 0.001$ and ${ }^{* * * *} P \leq 0.0001$. system. In brief, donor lung tissue was infected with SARS-CoV-2 and treated with apilimod or a positive control (remdesivir). Twenty-four hours after viral challenge, RNA was collected from cells and viral transcripts were quantified (Fig. $4 \mathrm{f}$ ). Supernatants were also processed at $24 \mathrm{~h}$ for quantification of viral titre by plaque assay (Fig. $4 \mathrm{~g}$ ).

The results reveal that apilimod potently antagonizes viral replication in tissues that reflect the primary site of SARS-CoV-2 replication. Apilimod is a specific PIKfyve kinase inhibitor, and was also found to inhibit viral replication during entry (Fig. 5a, b), consistent with observations that PIKfyve resides predominately in early endosomes and has an essential role in maintenance of endomembrane homeostasis ${ }^{47}$. Apilimod has been found to be well tolerated in humans, exhibiting a desirable safety profile at doses of up to $125 \mathrm{mg}$ twice daily ${ }^{2,48}$ and a peak serum concentration of $0.265 \pm 0.183 \mu \mathrm{M}$, indicating that therapeutic dosing may be achieved in patients at concentrations likely to promote antiviral activity. Apilimod has been evaluated in phase II clinical trials for the treatment of active Crohn's disease, rheumatoid arthritis and common variable immunodeficiency ${ }^{2,3}$, and in phase I studies for the treatment of follicular lymphoma ${ }^{49}$. Apilimod also efficiently inhibits Ebola virus, Lassa virus and Marburg virus in human cell lines, underscoring its potential broad-spectrum antiviral activity ${ }^{21,22}$. Evaluation of in vivo efficacy in suitable animal models will be highly instructive for the development of this molecule as a therapy for COVID-19.

\section{Discussion}

Since the beginning of January 2020, an extraordinary number of investigations and clinical trials have been initiated in a concerted effort to identify therapies against the rapidly growing COVID-19 pandemic. Critically, remdesivir was recently granted emergency use authorization (EUA) for the treatment of COVID-19 on the basis of data from a clinical trial conducted by the National Institute of Allergy and Infectious Diseases, which demonstrated significantly improved time to recovery $(47 \%)$ in treated patients ${ }^{10}$. However, this therapeutic end point is far from optimal, and the identification of additional candidate therapies would enable the development of combinatorial regimens, which would reflect the current treatment strategies for HIV-1 and hepatitis $\mathrm{C}$ virus ${ }^{50-52}$.

In this study, we report the high-throughput analysis of approximately 12,000 known drugs for activity against SARS-CoV-2 replication, revealing approximately 100 known drugs with antiviral activities against SARS-CoV-2 (Extended Data Fig. 10). On the basis of the known mechanisms of action of the compounds, we extrapolated a cellular map of druggable targets, pathways, biological processes and small molecules that modulate the SARS-CoV-2 replication cycle (Extended Data Fig. 5). Several major target classes were found to be enriched for activity in this analysis, including ion channels, G-protein-coupled receptors (GPCRs), proteases and kinases (Fig. 3a, Supplementary Table 3). It is important to note that selectivity and off-target activities of the identified compounds can vary, and thus observed antiviral activities may derive from either modulation of the annotated drug target or an off-target activity based on binding to a protein in the same or a divergent family. For example, we found that the activities of a RAR agonist could be reversed with the application of a RAR antagonist (Fig. 2b), but similar relationships could not be established for several GPCR agonists (Extended Data Fig. 3b). This is potentially 


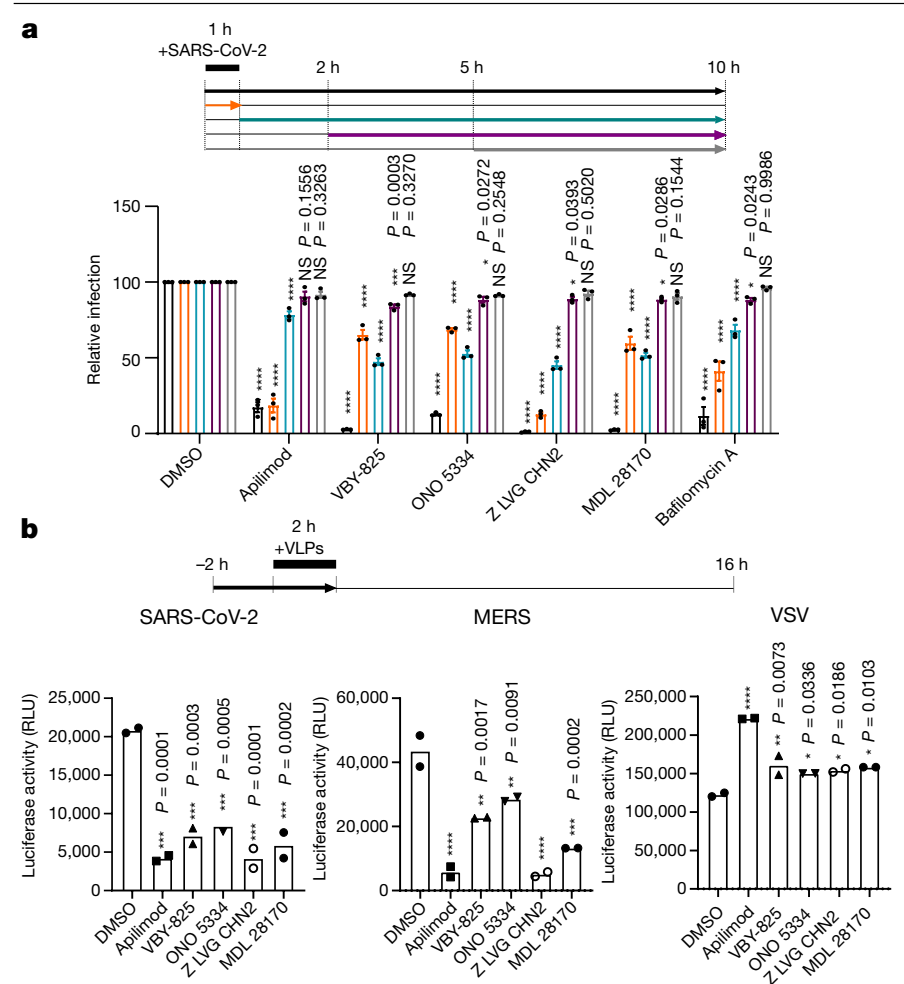

Fig. 5 | Apilimod and protease inhibitors block SARS-CoV-2 entry.

a, Time-of-addition assay. To synchronize infection, Vero E6 cells were infected for $1 \mathrm{~h}$ with SARS-CoV-2, and the inoculum was then removed. Cells were also incubated with the indicated compound at a concentration of $2.5 \mu \mathrm{M}$ at the indicated time points. Infection was quantified $10 \mathrm{~h}$ post-inoculation after fixation and staining for coronavirus NP. Data are normalized to the mean of DMSO-treated wells for each corresponding time point and are presented as mean \pm s.e.m. of $n=3$ independent experiments. Two-way ANOVA followed by Tukey post-test. Bafilomycin was used as a positive control.b, Virus-like particle (VLP) assay. Vero E6 cells were pre-treated for $2 \mathrm{~h}$ with the indicated compounds $(2.5 \mu \mathrm{M})$ and then infected for $2 \mathrm{~h}$ with SARS-CoV-2 (left), MERS (middle) or VSV (right) pseudotyped particles harbouring firefly luciferase (Methods). Inoculum was removed after a further $2 \mathrm{~h}$, and firefly luciferase signal was quantified $24 \mathrm{~h}$ post-inoculation. Data are mean \pm s.e.m. of $n=2$ independent experiments. One-way ANOVA followed by Dunnett posttest. ${ }^{*} P \leq 0.05,{ }^{* *} P \leq 0.01,{ }^{* * *} P \leq 0.001$ and ${ }^{* * * *} P \leq 0.0001$. RLU, relative light unit.

suggestive of off-target activities underlying the antiviral effects of some of these molecules.

We report the identification of 21 molecules, including remdesivir, which were confirmed to possess dose-activity relationships, and 13 of these compounds were found to have $\mathrm{EC}_{50}$ values lower than $500 \mathrm{nM}$ in at least one cell line (Figs. 3b, 4a, b, Extended Data Figs. 6c, 7). The pharmacokinetic properties of the individual compounds, including factors such as serum protein binding and bioavailability in the lung, will affect the potential for vivo antiviral efficacy. However, in conjunction with safety data from phase I multiple-ascending-dose studies, as well as reported peak serum concentrations in humans, these cellular potencies suggest that many of these drugs may harbour sufficient antiviral activity during therapeutic administration. To enable prioritization of known drugs for in vivo preclinical and clinical evaluation for the treatment of SARS-CoV-2, a summary of the publicly disclosed and relevant preclinical and clinical properties of the most advanced among these molecules are annotated in Supplementary Table 4. Thus, the availability of human safety and pharmacological data regarding clinical-stage molecules is expected to enable rapid preclinical and clinical assessment of these compounds. However, expedited regulatory review under EUA guidelines also provides a rationale for the development of earlier-stage candidate molecules that can be deployed for use during the current pandemic outbreak. It is critical that multiple therapeutic options that demonstrate efficacy against SARS-CoV-2 become available to mitigate potential emergence of drug resistance, and to enable the evaluation of optimal therapeutic cocktails that are broadly curative for COVID-19.

\section{Online content}

Any methods, additional references, Nature Research reporting summaries, source data, extended data, supplementary information, acknowledgements, peer review information; details of author contributions and competing interests; and statements of data and code availability are available at https://doi.org/10.1038/s41586-020-2577-1.

1. Yang, X. et al. Clinical course and outcomes of critically ill patients with SARS-CoV-2 pneumonia in Wuhan, China: a single-centered, retrospective, observational study. Lancet Respir. Med. 8, 475-481 (2020).

2. Sbrissa, D., Naisan, G., Ikonomov, O. C. \& Shisheva, A. Apilimod, a candidate anticancer therapeutic, arrests not only Ptdlns $(3,5) \mathrm{P}_{2}$ but also Ptdlns5P synthesis by PIKfyve and induces bafilomycin A1-reversible aberrant endomembrane dilation. PLOS ONE 13, e0204532 (2018).

3. Billich, A. Drug evaluation: apilimod, an oral IL-12/IL-23 inhibitor for the treatment of autoimmune diseases and common variable immunodeficiency. IDrugs 10, 53-59 (2007).

4. Cai, X. et al. PIKfyve, a class III PI kinase, is the target of the small molecular IL-12/IL-23 inhibitor apilimod and a player in Toll-like receptor signaling. Chem. Biol. 20, 912-921 (2013).

5. World_Health_Organization. Coronavirus Disease (COVID-19) Pandemic https://www.who. int/emergencies/diseases/novel-coronavirus-2019 (2020).

6. Chen, W. H., Strych, U., Hotez, P. J. \& Bottazzi, M. E. The SARS-CoV-2 vaccine pipeline: an overview. Curr. Trop. Med. Rep. 1-4, 00201-6 (2020).

7. Cascella, M., Rajnik, M., Cuomo, A., Dulebohn, S. C. \& Di Napoli, R. in StatPearls (StatPearls, 2020)

8. Chen, H. et al. First clinical study using HCV protease inhibitor danoprevir to treat naive and experienced COVID-19 patients. Preprint at medRxiv https://doi.org/10.1101/ 2020.03.22.20034041 (2020).

9. Warren, T. K. et al. Therapeutic efficacy of the small molecule GS-5734 against Ebola virus in rhesus monkeys. Nature 531, 381-385 (2016).

10. US Food and Drug Administration. FDA Issues Emergency Use Authorization for Potential COVID-19 Treatment https://www.fda.gov/news-events/press-announcements/corona virus-covid-19-update-fda-issues-emergency-use-authorization-potential-covid-19treatment (2020).

11. Kujawski, S. A. et al. First 12 patients with coronavirus disease 2019 (COVID-19) in the United States. Preprint at medRxiv https://doi.org/10.1101/2020.03.09.20032896 (2020)

12. Janes, J. et al. The ReFRAME library as a comprehensive drug repurposing library and its application to the treatment of cryptosporidiosis. Proc. Natl Acad. Sci. USA 115, 10750-10755 (2018).

13. Kim, Y. J. et al. The ReFRAME library as a comprehensive drug repurposing library to identify mammarenavirus inhibitors. Antiviral Res. 169, 104558 (2019).

14. Harbut, M. B. et al. Auranofin exerts broad-spectrum bactericidal activities by targeting thiol-redox homeostasis. Proc. Natl Acad. Sci. USA 112, 4453-4458 (2015).

15. Love, M. S. et al. A high-throughput phenotypic screen identifies clofazimine as a potential treatment for cryptosporidiosis. PLoS Negl. Trop. Dis. 11, e0005373 (2017).

16. Bollong, M. J. et al. Small molecule-mediated inhibition of myofibroblast transdifferentiation for the treatment of fibrosis. Proc. Natl Acad. Sci. USA 114, 4679-4684 (2017).

17. Li, Y. Y. \& Jones, S. J. Drug repositioning for personalized medicine. Genome Med. 4, 27 (2012).

18. Matsuyama, S. et al. Enhanced isolation of SARS-CoV-2 by TMPRSS2-expressing cells. Proc. Natl Acad. Sci. USA 117, 7001-7003 (2020)

19. Park, W. B. et al. Virus Isolation from the first patient with SARS-CoV-2 in Korea. J. Korean Med. Sci. 35, e84 (2020).

20. To, K. K. et al. Consistent detection of 2019 novel coronavirus in saliva. Clin. Infect. Dis. 71, 841-843 (2020).

21. Nelson, E. A. et al. The phosphatidylinositol-3-phosphate 5-kinase inhibitor apilimod blocks filoviral entry and infection. PLoS Negl. Trop. Dis. 11, e0005540 (2017).

22. Qiu, S. et al. Ebola virus requires phosphatidylinositol $(3,5)$ bisphosphate production for efficient viral entry. Virology 513, 17-28 (2018).

23. Yamamoto, N. et al. HIV protease inhibitor nelfinavir inhibits replication of SARS-associated coronavirus. Biochem. Biophys. Res. Commun. 318, 719-725 (2004).

24. Shweta, C., Yashpal S., M. \& Shailly, T. Identification of SARS-CoV-2 cell entry inhibitors by drug repurposing using in silico structure-based virtual screening approach. Front. Immunol. 11, 1664 (2020).

25. Xu, Z. et al. Nelfinavir is active against SARS-CoV-2 in Vero E6 cells. Preprint at https:// doi.org/10.26434/chemrxiv.12039888.v1 (2020).

26. Wang, S. Q. et al. Virtual screening for finding natural inhibitor against cathepsin- $L$ for SARS therapy. Amino Acids 33, 129-135 (2007).

27. Schneider, M. et al. Severe acute respiratory syndrome coronavirus replication is severely impaired by MG132 due to proteasome-independent inhibition of M-calpain. J. Virol. 86, 10112 (2012). 
28. Subramanian, A. et al. Gene set enrichment analysis: a knowledge-based approach for interpreting genome-wide expression profiles. Proc. Natl Acad. Sci. USA 102, 15545-15550 (2005).

29. Mootha, V. K. et al. PGC-1a-responsive genes involved in oxidative phosphorylation are coordinately downregulated in human diabetes. Nat. Genet. 34, 267-273 (2003).

30. Butler, D. J. et al. Host, viral, and environmental transcriptome profiles of the severe acute respiratory syndrome coronavirus 2 (SARS-CoV-2). Preprint at bioRxiv https://doi.org/ 10.1101/2020.04.20.048066 (2020).

31. Deprez, M. et al. A single-cell atlas of the human healthy airways. Am. J. Respir. Crit. Care Med. 34, https://doi.org/10.1164/rccm.201911-21990C (2020).

32. Kwan, C. Y. \& Achike, F. I. Tetrandrine and related bis-benzylisoquinoline alkaloids from medicinal herbs: cardiovascular effects and mechanisms of action. Acta Pharmacol. Sin 23, 1057-1068 (2002).

33. Sakurai, Y. et al. Two-pore channels control Ebola virus host cell entry and are drug targets for disease treatment. Science 347, 995-998 (2015).

34. Inciardi, R. M. et al. Cardiac involvement in a patient with coronavirus disease 2019 (COVID-19). JAMA Cardiol. 5, 819-824 (2020).

35. Kapoor, A. et al. Cardiovascular risks of hydroxychloroquine in treatment and prophylaxis of COVID-19 patients: a scientific statement from the Indian Heart Rhythm Society. Indian Pacing Electrophysiol. J. 20, 117-120 (2020).

36. D'Alessandro, S. et al. The use of antimalarial drugs against viral infection. Microorganisms 8, E85 (2020).

37. Lucile White, E. et al. A TIBO derivative, R82913, is a potent inhibitor of HIV-1 reverse transcriptase with heteropolymer templates. Antiviral Res. 16, 257-266 (1991).

38. De Wit, S. et al. Pharmacokinetics of R 82913 in AIDS patients: a phase I dose-finding study of oral administration compared with intravenous infusion. Antimicrob. Agents Chemother. 36, 2661-2663 (1992).

39. Garrelts, J. C. Clofazimine: a review of its use in leprosy and Mycobacterium avium complex infection. DICP 25, 525-531 (1991).

40. Björck, L., Grubb, A. \& Kjellén, L. Cystatin C, a human proteinase inhibitor, blocks replication of herpes simplex virus. J. Virol. 64, 941-943 (1990).

41. Zhou, Y. \& Simmons, G. Development of novel entry inhibitors targeting emerging viruses. Expert Rev. Anti Infect. Ther. 10, 1129-1138 (2012).

42. Mori, Y. et al. Processing of capsid protein by cathepsin L plays a crucial role in replication of Japanese encephalitis virus in neural and macrophage cells. J. Virol. 81, 8477-8487 (2007).

43. Simmons, G. et al. Inhibitors of cathepsin $L$ prevent severe acute respiratory syndrome coronavirus entry. Proc. Natl Acad. Sci. USA 102, 11876-11881 (2005).

44. Marzi, A., Reinheckel, T. \& Feldmann, H. Cathepsin B \& L are not required for ebola virus replication. PLoS Negl. Trop. Dis. 6, e1923 (2012).

45. Eastell, R. et al. Safety and efficacy of the cathepsin K inhibitor ONO-5334 in postmenopausal osteoporosis: the OCEAN study. J. Bone Miner. Res. 26, 1303-1312 (2011).

46. Eastell, R. et al. Effect of ONO-5334 on bone mineral density and biochemical markers of bone turnover in postmenopausal osteoporosis: 2-year results from the OCEAN study. J. Bone Miner. Res. 29, 458-466 (2014).

47. Rutherford, A. C. et al. The mammalian phosphatidylinositol 3-phosphate 5-kinase (PIKfyve) regulates endosome-to-TGN retrograde transport. J. Cell Sci. 119, 3944-3957 (2006).

48. Gayle, S. et al. Identification of apilimod as a first-in-class PIKfyve kinase inhibitor for treatment of B-cell non-Hodgkin lymphoma. Blood 129, 1768-1778 (2017).
49. Sultana, F. et al. Snx10 and PIKfyve are required for lysosome formation in osteoclasts. J. Cell. Biochem. 121, 2927-2937 (2020).

50. Matthew, A. N., Kurt Yilmaz, N. \& Schiffer, C. A. Mavyret: A pan-genotypic combination therapy for the treatment of hepatitis C infection. Biochemistry 57, 481-482 (2018).

51. Ferenci, P. New anti-HCV drug combinations: who will benefit? Lancet Infect. Dis. 17, 1008-1009 (2017).

52. Cihlar, T. \& Fordyce, M. Current status and prospects of HIV treatment. Curr. Opin. Virol. 18, 50-56 (2016)

Publisher's note Springer Nature remains neutral with regard to jurisdictional claims in published maps and institutional affiliations.

(c) The Author(s), under exclusive licence to Springer Nature Limited 2020

${ }^{1}$ Immunity and Pathogenesis Program, Infectious and Inflammatory Disease Center, Sanford Burnham Prebys Medical Discovery Institute, La Jolla, CA, USA. ${ }^{2}$ State Key Laboratory of Emerging Infectious Diseases, Li Ka Shing Faculty of Medicine, The University of Hong Kong, Hong Kong Special Administrative Region, Hong Kong, China. ${ }^{3}$ Department of Microbiology, Li Ka Shing Faculty of Medicine, The University of Hong Kong, Hong Kong Special Administrative Region, Hong Kong, China. ${ }^{4}$ Carol Yu Centre for Infection, Li Ka Shing Faculty of Medicine, The University of Hong Kong, Hong Kong Special Administrative Region, Hong Kong, China. ${ }^{5}$ Center for Integrative Bioinformatics Vienna, Max Perutz Laboratories, University of Vienna and Medical University of Vienna, Vienna, Austria. ${ }^{6} \mathrm{Calibr}$ at Scripps Research, La Jolla, CA, USA. ${ }^{7}$ Department of Medicine, University of California, San Diego, La Jolla, CA, USA. ${ }^{8}$ Cancer Data Science Laboratory, Center for Cancer Research, National Cancer Institute, National Institute of Health, Bethesda, MD, USA. ${ }^{9}$ Biological Sciences Graduate Program, University of Maryland, College Park, MD, USA. ${ }^{10}$ Department of Microbiology, Icahn School of Medicine at Mount Sinai, New York, NY, USA. ${ }^{11}$ Global Health and Emerging Pathogens Institute, Icahn School of Medicine at Mount Sinai, New York, NY, USA. ${ }^{12}$ Graduate School of Biomedical Sciences, Icahn School of Medicine at Mount Sinai, New York, New York, USA. ${ }^{13}$ Huffington Foundation Center for Cell-based Research in Parkinson's Disease, Department for Cell, Regenerative and Developmental Biology, Black Family Stem Cell Institute, Icahn School of Medicine at Mount Sinai, New York, NY, USA. ${ }^{14}$ Department of Surgery, Li Ka Shing Faculty of Medicine, The University of Hong Kong, Hong Kong Special Administrative Region, Hong Kong, China. ${ }^{15}$ Texas Biomedical Research Institute, San Antonio, TX, USA. ${ }^{16}$ Department of Biological Sciences, Purdue University, West Lafayette, IN, USA. ${ }^{17}$ Department of Biochemistry, Purdue University, West Lafayette, IN, USA. ${ }^{18}$ Inception Therapeutics, San Diego, CA, USA. ${ }^{19}$ Department of Molecular and Medical Pharmacology, University of California, Los Angeles, CA, USA. ${ }^{20}$ Department of Integrative, Structural and Computational Biology, The Scripps Research Institute, La Jolla, CA, USA. ${ }^{21}$ Department of Medicine, Division of Infectious Diseases, Icahn School of Medicine at Mount Sinai, New York, NY, USA. ${ }^{22}$ The Tisch Cancer Institute, Icahn School of Medicine at Mount Sinai, New York, NY, USA. ${ }^{23}$ These authors contributed equally: Laura Riva, Shuofeng Yuan. ${ }^{\bowtie}$ e-mail: achatterjee@scripps.edu; kyyuen@hku.hk; schanda@sbpdiscovery.org 


\section{Methods}

\section{Data reporting}

No statistical methods were used to predetermine sample size. Compounds were spotted in a randomized order on the plates during the primary screen. All the other experiments were not randomized. Investigators were blinded to allocation during the primary screen and the corresponding orthogonal validation, during both assay performance and outcome assessment. For all the other assays, the investigators were not blinded.

\section{Cells and viruses}

The SARS-CoV-2 HKU-001a strain was isolated from the nasopharyngeal aspirate specimen from a patient with laboratory-confirmed COVID-19 in Hong Kong ${ }^{20}$. The nasopharyngeal aspirate specimen was inoculated on Vero E6 cells (ATCC CRL-1586). The inoculated cells were monitored daily for cytopathic effects by light microscopy and the cell supernatants were collected daily for quantitative PCR with reverse transcription (RT- $\mathrm{qPCR}$ ) to assess the viral load. Extensive cytopathic effects were observed at $72 \mathrm{~h}$ post-inoculation (hpi) and positive SARS-CoV-2 replication was confirmed by RT-qPCR using specific primers and probes against SARS-CoV-2. Whole-genome sequencing for the SARS-CoV-2 isolate was done using a MinION device (Oxford Nanopore Technologies) supplemented by Sanger sequencing, as previously described ${ }^{53}$. SARS-CoV-2 HKU-001a (GenBank accession number:MT230904) was propagated and titrated in VeroE6 cells using plaque assays. The virus was passaged three times before being used for the experiments ${ }^{54}$. The SARS-CoV-2 USA-WA1/2020 strain, isolated from an oropharyngeal swab from a patient with a respiratory illness who developed clinical disease (COVID-19) in January 2020 in Washington, USA, was obtained from BEI Resources (NR-52281). The virus was inoculated on Vero E6 cells transfected with exogenous human ACE2 and TMPRSS2, collected after one passage and stored at $-80^{\circ} \mathrm{C}$ in aliquots. PFU and median tissue culture infectious dose ( TCID $\left._{50}\right)$ assays were performed to titrate the cultured virus. Vero E6 cells were maintained in Dulbecco's modified eagle medium (DMEM, Gibco) supplemented with $10 \%$ heat-inactivated fetal bovine serum (FBS, Gibco), $50 \mathrm{U} \mathrm{ml}^{-1}$ penicillin, $50 \mathrm{\mu g} \mathrm{ml}^{-1}$ streptomycin, $1 \mathrm{mM}$ sodium pyruvate (Gibco), $10 \mathrm{mM}$ 4-(2-hydroxyethyl)-1-piperazineethanesulfonic acid (HEPES, Gibco), and $1 \times$ MEM non-essential amino acids solution (Gibco). Huh-7 and HEK293T cells stably expressing ACE2 (Huh-7-hACE2 and HEK293T-hACE2) were generated by transducing Huh-7 (Apath) and HEK293T (ATCCCRL-3216) cells with ACE2-expressing lentivirus, followed by selection of resistant cells with puromycin (InvivoGen) at $2 \mu \mathrm{g} \mathrm{ml}^{-1}$ for 14 days. The resistant cells were maintained in DMEM (Gibco) supplemented with $10 \%$ heat-inactivated FBS (Gibco), $50 \mathrm{U} \mathrm{ml}^{-1}$ penicillin, $50 \mu \mathrm{g} \mathrm{ml}^{-1}$ streptomycin, and $1 \mu \mathrm{g} \mathrm{ml}^{-1}$ puromycin. The expression of ACE2 in these ACE2 stable cell lines was determined by western blot analysis. BHK-21/WI-2 cells (Kerafast) were maintained in DMEM (Gibco) supplemented with $10 \%$ heat-inactivated FBS (Gibco), $50 \mathrm{U} \mathrm{ml}^{-1}$ penicillin, $50 \mathrm{\mu g} \mathrm{ml}^{-1}$ streptomycin. Cell lines were ordered directly from the distributors and not authenticated. All cells were tested negative for mycoplasma contamination, except for Huh-7-ACE2 cells. All experiments involving live SARS-CoV-2 followed the approved standard operating procedures of the biosafety level 3 facility at the University of Hong Kong ${ }^{55}$ and Sanford Burnham Prebys Medical Discovery Institute.

\section{Chemical libraries}

The LOPAC1280 library is a collection of 1,280 pharmacologically active compounds, covering all the major target classes, including kinases, GPCRs, neurotransmission and gene regulation (Sigma). The ReFRAME library ${ }^{12}$, built at Calibr, contains approximately 12,000 high-value molecules assembled by combining three databases (Clarivate Integrity, GVK Excelra GoStar and Citeline Pharmaprojects) for fast-track drug discovery. This library contains US Food and Drug Administration
(FDA)-approved and registered drugs (approximately 35\%), investigational new drugs (approximately 58\%) and preclinical compounds (approximately 3\%).

\section{Drug screening}

Compounds from the LOPAC1280 and ReFRAME libraries were transferred into F-BOTTOM, $\mu$ CLEAR, BLACK 384-well plates (Greiner) using an Echo 550 Liquid Handler (Labcyte). All compounds were diluted in culture media to a final concentration of $5 \mu \mathrm{M}$ during screening. In brief, Vero E6 cells were seeded in 384-well plates, on top of pre-spotted compounds, at a density of 3,000 cells per well in $40 \mu$ l using a microFlo select dispenser (BioTek Instruments). Sixteen hours after seeding, the cells were infected by adding $10 \mu$ of SARS-CoV-2 HKU-001a per well at an MOI of 0.01. CPE was indirectly quantified as the presence of ATP in live cells by using the CellTiter-Glo (Promega) luminescent cell viability assay at $72 \mathrm{hpi}$. Data were normalized to the median of each plate. For the ReFRAME library, the Z-score was calculated on the basis of the $\log _{2}$ (fold change) $\left(\log _{2} \mathrm{FC}\right)$ with the average and standard deviation of each plate. The screen was performed in duplicate by running the assay in parallel for the LOPAC1280 library or as two independent experiments for the ReFRAME collection. Twenty-eight compounds from the LOPAC1280 library were selected according to the cutoff of $>5 \times$ s.d. of $\log _{2} \mathrm{FC}$ and included in a dose-response confirmation assay. Compounds from the ReFRAME collection were ranked according to their $Z$-score. The top 100 hits from each replicate were selected ( 25 overlapping). Seventy-five additional hits were chosen according to their ranking based on the average $Z$-score. The last 48 hits were selected according to drug target and pathway enrichment analysis. The 298 prioritized hits were included in a dose-response confirmation assay.

\section{Immunofluorescence assay and quantification of SARS-CoV-2 infection}

At several points throughout experimentation, infected Vero E6 and human HEK293T-ACE2 or Huh-7-ACE2 cells were subjected to orthogonal validation using an immunofluorescence-based imaging assay, labelling the viral NP in infected cells. In each assay detailed below, including dose-response assays, time-of-addition assay and drug synergy quantification assay, infected cells were fixed at the indicated time post-infection with 5\% paraformaldehyde for $4 \mathrm{~h}$ and permeabilized with $0.5 \%$ Triton X-100 for 5 min. After blocking with 3\% bovine serum albumin (BSA) for $30 \mathrm{~min}$, the cells were incubated for $1 \mathrm{~h}$ at room temperature with rabbit anti-SARS-CoV NP serum, which exhibits strong cross-reactivity with SARS-CoV-2 NP. After two washes with phosphate-buffered saline (PBS), the cells were incubated with Alexa Fluor 488-conjugated goat-anti-rabbit IgG (Thermo Fisher Scientific) for $1 \mathrm{~h}$ at room temperature. After two additional washes, PBS supplemented with $0.1 \mu \mathrm{g} \mathrm{ml}^{-1}$ DAPI (BioLegend) was added to the cells for at least $30 \mathrm{~min}$ before imaging. Images were acquired using the Celigo Image Cytometer (Nexcelom). The assay results and data analysis enabled us to determine infectivity and viability or cytotoxicity. On the basis of all infectivity and cytotoxicity values, a four-parameter logistic nonlinear regression model was used to calculate $\mathrm{EC}_{50}$ and 50\% cytotoxic concentration $\left(\mathrm{CC}_{50}\right)$ values whenever required.

\section{High-throughput orthogonal validation of the primary hits and potency evaluation}

The selected hits were further validated by immunofluorescence in an eight-point dose-response experiment to determine $\mathrm{EC}_{50}$ and $\mathrm{CC}_{50}$ concentration values. In brief, 3,000 Vero E6 cells were added into 384-well plates pre-spotted with compounds, in a volume of $40 \mu \mathrm{l}$. The final concentration of compound ranged from $1.1 \mathrm{nM}$ to $2.5 \mu \mathrm{M}$. Sixteen hours post-seeding, $10 \mu$ l of SARS-CoV-2 USA-WA1/2020 was added to each well, at an MOI of 0.01. Twenty-four hours post-infection, cells were fixed and subjected to a cell-based high-content imaging assay to detect SARS-CoV-2 NP within infected cells, as described in the 
'Immunofluorescence assay and quantification of SARS-CoV-2 infection' section.

\section{Enrichment analysis}

Compounds were annotated in the three databases used to assemble the ReFRAME library (Clarivate Integrity, GVK Excelra GoStar and Citeline Pharmaprojects (Informa MOA)) according to a variety of properties, including targets, pathways, indications and mechanisms of actions (MOA). Each annotation property was tested for enrichment among the screening hits using the GSEA software ${ }^{28,29}$. The compounds annotated for each property were treated as a gene set. For each set of vendor annotations, the background compound set was defined as the set of compounds annotated for any property by that vendor. Enrichment results at $P<0.05$ and FDR $q<0.33$ were defined as significant. Additional enrichment analyses were performed using the free online meta-analysis tool Metascape ${ }^{56} . P$ values were generated using a one-sided hypergeometric test ${ }^{56}$. Values were corrected for multiple hypothesis testing by the Benjamini-Hochberg method ${ }^{56}$.

\section{Gene-expression analysis}

Vero E6 cells were either mock-infected or infected with SARS-CoV-2 USA-WA1/2020 (MOI = 0.3). Twenty-four hours after infection, cells were collected and total RNA was extracted using the Qiagen RNeasy Plus Mini Kit. Three replicates were performed for each group, resulting in a total of six samples. The quality of the extracted RNA was assessed with the Agilent 2100 Bioanalyzer. Libraries were prepared from total RNA following ribosome RNA depletion using the standard protocol according to Illumina. Total RNA sequencing was then performed on the Illumina NextSeq system; 150 bp paired-end runs were performed and 100 million raw reads per sample were generated (GEO accession number: GSE153940). STAR ${ }^{57}$ was used to align the reads to reference the genome of the African green monkey (Chlorocebussabaeus, https://useast.ensembl.org/Chlorocebus_sabaeus/Info/ Annotation), with the SARS-CoV-2 genome (https://www.ncbi.nlm. nih.gov/nuccore/NC_045512), selected as the reference genome. The R package DESeq $2^{58}$ was used for differential expression (DE) analysis between the virus-infected and the control samples. $P$ values (pval) were computed by DESeq 2 with generalized linear models testing for the difference in log-transformed expression values between control and virus-infected samples (that is, they are essentially two-sided $P$ values); adjusted $P$ values (padj) were computed with the BenjaminiHochberg method. GSEA analysis ${ }^{28}$ was performed on the DE results using Kyoto Encyclopedia of Genes and Genomes (KEGG) and Reactome pathway annotations obtained from the MSigDB database ${ }^{59}$, using the R package fgsea. Empirical $P$ values (pval) were computed by fgsea using a permutation test (two-sided), and were adjusted (padj) using the Benjamini-Hochberg method. Specifically, this step was performed to check whether the enriched drug-targeting pathways as given in Extended Data Fig. 3a showed significant enrichment by the GSEA analysis in the virus-infected samples compared to the control. Gene-expression analysis on human data was based on a publicly available RNA-seq dataset of nasopharyngeal swab specimens taken from patients infected with SARS-CoV- $2^{30}$ and a publicly available single-cell RNA-seq dataset consisting of profiled samples from four macro-anatomical locations of human airway epithelium in healthy living volunteers ${ }^{31}$ (Extended Data Fig. 4). We used the pre-calculated raw gene counts and inferred cell types from this dataset. For each gene, the fraction of cells with non-zero expression values was calculated in nasal, tracheal, intermediate and distal samples from multiple donors. Values for each sampling location were averaged across donors. To analyse gene-expression levels in different cell types, the fractions of cells with non-zero expression values were determined in all cells of a given cell type across samples. Cell types with a total of less than 250 cells detected were excluded from the analysis. Clustered heat maps were generated in $\mathrm{R}$ using the pheatmap and viridis packages.

\section{Time-of-addition assay}

Twenty-thousand Vero E6 cells were seeded in 96-well plates. The following day, cells were infected with SARS-CoV-2 USA-WA1/2020 $(\mathrm{MOI}=1.5)$. After $1 \mathrm{~h}$, the viral inoculum was removed and cells were washed with ice-cold PBS, before addition of fresh medium. DMSO vehicle or $2.5 \mu \mathrm{M}$ of the indicated compound was added at different time points, according to the timeline illustrated in Fig. 5a. Cells were fixed at $10 \mathrm{~h}$ post-infection and subjected to an immunofluorescence assay targeting SARS-CoV-2 NP, in order to quantify the percentage of infected cells, as described in the Immunofluorescence assay and quantification of SARS-CoV-2 infection' section.

\section{Pseudotyping of VSV and pseudotype-based inhibition assay}

VSV pseudotyped with S proteins of MERS and SARS-CoV-2 were generated according to a published protocol ${ }^{60}$. In brief, BHK-21/WI-2 cells (Kerafast) transfected to express the $S$ proteins were inoculated with VSV-G pseudotyped $\Delta \mathrm{G}$-luciferase VSV (Kerafast). After a 2-h incubation at $37^{\circ} \mathrm{C}$, the inoculum was removed and DMEM supplemented with $5 \%$ FBS, $50 \mathrm{U} \mathrm{ml}^{-1}$ penicillin and $50 \mu \mathrm{g} \mathrm{ml}^{-1}$ streptomycin was added back to cells. Pseudotyped particles were collected $24 \mathrm{~h}$ post-inoculation, then centrifuged at $1,320 \mathrm{~g}$ to remove cell debris and stored at $-80^{\circ} \mathrm{C}$ until use. To determine the effect of the selected compounds on viral entry, Vero E6 cells were treated with each compound at a concentration of $2.5 \mu \mathrm{M}$ for $1 \mathrm{~h}$ before inoculation with respective pseudotyped VSV. After $2 \mathrm{~h}$ inoculation in the presence of the compounds, the inoculum was removed, and fresh medium was added to cells for further culture. The activity of firefly luciferase as a readout of infected cells was measured using the bright-Glo luciferase assay (Promega) for quantitative determination at $16 \mathrm{hpi}$.

\section{Inhibition of SARS-CoV-2 PLpro and main protease by compounds}

SARS-CoV-2 (GenBank accession number: MN908947) PLpro (polyprotein residues 1564-1874) and main protease (Mpro) (polyprotein residues 3259-3569) were expressed and purified from Escherichia coli according to established methods ${ }^{61}$. In brief, PLpro was expressed with a $\mathrm{N}$-terminal $\mathrm{His}_{6}$ tag and purified by $\left(\mathrm{Ni}^{2+}\right)$-affinity chromatography. The $\mathrm{His}_{6}$ tag was removed by Tobacco Etch Virus (TEV) protease digestion and then passed over a size-exclusion chromatography column for a final purification step. Mpro was also expressed with a $\mathrm{N}$-terminal $\mathrm{His}_{6}$ tag that is removed during expression by an Mpro-catalysed autocleavage reaction. Mpro was purified by a combination of anion-exchange, hydrophobic interaction and size-exclusion chromatographic steps. SARS-CoV-2 PLpro enzyme inhibition assays were performed in triplicate in Costar 96-well black microplates using the peptide substrate Arg-Leu-Arg-Gly-Gly-AMC (RLRGG-AMC) at a final concentration of $50 \mu \mathrm{M}$, which is well below the Michaelis $\left(K_{\mathrm{m}}\right)$ value for this substrate $(>1 \mathrm{mM})$. Assays were performed in buffer composed of $50 \mathrm{mM} \mathrm{HEPES}$, $\mathrm{pH} 7.5,0.1 \mathrm{mg} \mathrm{ml}^{-1} \mathrm{BSA}, 5 \mathrm{mM}$ DTT and $50 \mu \mathrm{M}$ RLRGG-AMC substrate in a final assay volume of $100 \mu \mathrm{l}$. Selected compounds were included in the assays at varying concentrations ranging from 1 to $50 \mu \mathrm{M}$. Compounds GRL-0617 and 3k, known SARS-CoV PLpro inhibitors ${ }^{62,63}$, were used at a concentration of $50 \mu \mathrm{M}$ as control inhibitors of SARS-CoV-2. Each of these compounds inhibits SARS-CoV-2 at 95 to $100 \%$ at this concentration. The final concentration of DMSO after the addition of compounds at all concentrations is $1 \%$. Enzyme reactions were initiated with enzyme (final concentration of $\sim 150 \mathrm{nM}$ ) and product formation was monitored over time at an emission wavelength of $460 \mathrm{~nm}$ with an excitation wavelength of $360 \mathrm{~nm}$ using a CLARIOstar Plus Microplate Reader. Enzyme activity in the absence (zero per cent inhibition control) and presence of compounds were used to calculate the per cent inhibition at each compound concentration. SARS-CoV-2 Mpro enzyme inhibition assays were performed in triplicate in Costar 3694 EIA/RIA 96-well half-area, flat bottom plates using the UIVT-3 peptide 
substrate (HiLyte Fluor $_{488}$-ESATLQSGLRKAK- $\mathrm{QXL}_{520}-\mathrm{NH}_{2}$ ) that was custom synthesized by Anaspec. The final concentration of substrate used was $2 \mu \mathrm{M}$ which is well below the $K_{\mathrm{m}}$ of this substrate $(>250 \mu \mathrm{M})$. Mpro assays were performed in assay buffer (50 mM HEPES pH 7.50, $0.1 \mathrm{mg} \mathrm{ml}^{-1} \mathrm{BSA}, 0.01 \%$ Triton X-100, 2 mM DTT, $1 \%$ DMSO) by preincubating enzyme at a final concentration of $200 \mathrm{nM}$ with inhibitor (1 to $50 \mu \mathrm{M}$ ) for $20 \mathrm{~min}$. After this time, the reaction was initiated by adding $20 \mu \mathrm{l}$ of the UIVT3 substrate. The increase in fluorescence intensity was measured over time at an emission wavelength of $530 \mathrm{~nm}$ with an excitation wavelength of $485 \mathrm{~nm}$ using a CLARIOstar Plus Microplate Reader. Compound 10 , a potent inhibitor of SARS Mpro ${ }^{64}$, was used as a control inhibitor of SARS-CoV-2 at a concentration of $50 \mu \mathrm{M}$ (data not shown). This compound inhibits SARS-CoV-2 at 95 to $100 \%$ under these reaction conditions. Enzyme activity in the absence (zero per cent inhibition control) and presence of compounds were used to calculate the per cent inhibition at each compound concentration.

\section{Drug synergy quantification}

Four-thousand Vero E6 cells were seeded in 384-well plates pre-spotted with indicated compounds, concentration ranging from $1 \mathrm{nM}$ to $2.5 \mu \mathrm{M}$ in a dose-response manner. Either DMSO vehicle or remdesivir ( $320 \mathrm{nM}$, $800 \mathrm{nM}$ or $1,200 \mathrm{nM}$ ) was added to the medium. Sixteen hours later, cells were infected with SARS-CoV-2 USA-WA1/2020 (MOI=0.1). Twenty-four hours post-infection, cells were fixed and an immune-fluorescence assay targeting SARS-CoV-2 NP was performed as described in the 'Immunofluorescence assay and quantification of SARS-CoV-2 infection' section. Synergy of drug combinations was assessed using the Bliss independence model ${ }^{65}$, which predicts that if two drugs, DA and $\mathrm{DB}$, with experimentally determined fractional effects $f_{\mathrm{A}}$ and $f_{\mathrm{B}}$ have an additive effect, their expected fractional combinatorial effect is $f_{\mathrm{AB}}=f_{\mathrm{A}}+f_{\mathrm{B}}-\left(f_{\mathrm{A}} \times f_{\mathrm{B}}\right)$.

\section{Validation of antiviral activity in human cell lines}

Six-thousand ACE2-transduced HEK293T cells and six-thousand ACE2-transduced Huh-7 cells were seeded in 384-well plates, pre-spotted with increasing doses (final concentration ranging from $1 \mathrm{nM}$ to $2.5 \mu \mathrm{M}$ ) (Fig. 4a, b, Extended Data Fig. 7) of each compound. After $16 \mathrm{~h}$, cells were infected with SARS-CoV-2 USA-WA1/2020 (MOI $=0.3$ and 0.2 for HEK293T-ACE2 and Huh-7-ACE2 cells, respectively). Twenty-four hours post-infection, cells were fixed with $5 \%$ paraformaldehyde and an immunofluorescence assay detecting SARS-CoV-2 NP was performed, as described in the Immunofluorescence assay and quantification of SARS-CoV-2 infection' section.

\section{Validation of antiviral activity in human iPSC-derived pneumocyte-like cells}

Human embryonic stem cell lines hPSC1 (H9,WiCell) and hPSC2 (Lis38-derived, a gift from J. Hanna, ISM ESCRO Project no. 14-005) were cultured with mTeSR (Stemcell Technologies, 85850) on Vitronectin XF (Stemcell Technologies, 07180)-coated tissue culture plates and split in a ratio of 1:6 to 1:12 every 4-6 days with Versene (Life Technologies, 15040066 ). When cells were $70-80 \%$ confluent, they were collected with Gentle dissociation reagent (Stemcell Technologies, 07174 ) and $2 \times 10^{6}$ cells per $10 \mathrm{~cm}^{2}$ were plated on Vitronectin-coated tissue culture plates in mTeSR. Definitive endoderm differentiation was induced following the described protocol ${ }^{66}$. Cells were split after 4 days and maturated for 6 more days or further induced to differentiate based on an adapted alveolar differentiation protocol ${ }^{67}$ in Iscove's modified Dulbecco's medium (IMDM, Life Technologies, 31980030) supplemented with 10\% FBS (Sigma, F4135), 2 mM L-glutamine (Life Technologies 25030081), 0.5 4 M all-trans-retinoic acid (Sigma, R2626), $10 \mathrm{ng} \mathrm{ml}^{-1}$ FGF-10 (R\&D Systems, 345-FG-025), $10 \mathrm{ng} \mathrm{ml}^{-1}$ EGF (R\&D Systems, 236-EG-01M), $100 \mathrm{ng} \mathrm{ml}^{-1} \mathrm{Wnt} 3 \mathrm{a}$ (R\&D Systems, 5036-WN010), $10 \mathrm{ng} \mathrm{ml}^{-1} \mathrm{KGF}$ (R\&D Systems, 251-KG-050) and $5 \mathrm{ng} \mathrm{ml}^{-1} \mathrm{BMP}-4$ (R\&D Systems, 314-BP-010). Viral infections were performed on day
11 of differentiation. DMSO or the indicated compound was added to the medium two hours before infection. ONO-5334 and MDL28170 were tested with hPSC1; apilimod was tested with hPSC2 cells at stage 1 or 2 , respectively. Cells were then infected by inoculation with $1 \times 10^{5}$ PFU of SARS-CoV-2USA-WA1/2020. Two days post-infection, cells were collected for flow cytometry (CoV-NP staining) quantifications. The gating strategy is described in the Supplementary Fig. 1. An MTT assay was also performed on non-infected samples, in order to assess the cytotoxicity of the compounds.

Validation of antiviral activity in human ex vivo lung tissues Human lung tissues for ex vivo studies were obtained from patients undergoing surgical operations at Queen Mary Hospital, Hong Kong as previously described ${ }^{68}$. The donors gave written consent as approved by the Institutional Review Board of the University of Hong Kong/Hospital Authority Hong Kong West Cluster (UW13-364). The freshly obtained lung tissues were processed into small rectangular pieces and were rinsed with advanced DMEM/F12 medium (Gibco, Thermo Fisher Scientific) supplemented with $2 \mathrm{mM}$ of HEPES (Gibco), $1 \times$ GlutaMAX (Gibco), $100 \mathrm{U} \mathrm{ml}^{-1}$ penicillin and $100 \mathrm{\mu g} \mathrm{ml}^{-1}$ streptomycin. The specimens were infected with SARS-CoV-2 HKU-001a with an inoculum of $1 \times 10^{6} \mathrm{PFU} \mathrm{ml}^{-1}$ at $500 \mu \mathrm{l}$ per well. After $2 \mathrm{~h}$, the inoculum was removed, and the specimens were washed 3 times with PBS. The infected human lung tissues were then cultured in $1 \mathrm{ml}$ of advanced DMEM/F12 medium with $2 \mathrm{mM}$

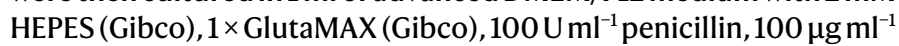
streptomycin, $20 \mu \mathrm{g} \mathrm{ml}^{-1}$ vancomycin, $20 \mu \mathrm{g} \mathrm{ml}^{-1}$ ciprofloxacin, $50 \mu \mathrm{g} \mathrm{ml}^{-1}$ amikacin, and $50 \mu \mathrm{g} \mathrm{ml}^{-1}$ nystatin. Supernatants were collected at $24 \mathrm{hpi}$ for plaque assays. The lung tissues were collected at 24 hpi in RLT buffer (Qiagen) with DTT (Qiagen) for RT-qPCR analysis of viral load and normalized against human $G A P D H$.

\section{Reporting summary}

Further information on research design is available in the Nature Research Reporting Summary linked to this paper.

\section{Data availability}

Additional data referred to in the text are available in Supplementary Tables 1-4, and from https://reframedb.org (assay A00440). Complete sequences of SARS-CoV-2 HKU-001a and SARS-CoV-2 USA-WA1/2020 are available through GenBank (accession numbers MT230904 (HKU-001a), MT246667 and MN908947 (USA-WA1/2020)). RNA-seq data in Supplementary Table 2 were aligned with the genome of the African green monkey (Chlorocebus sabaeus, https://uswest.ensembl. org/Chlorocebus_sabaeus/Info/Annotation) and with the SARS-CoV-2 genome (NCBI nucleotide sequence NC045512) selected as the reference genome. The dataset is available on Gene Expression Omnibus (GEO) with accession number GSE153940. Extended Data Figure 4 is based on analysis of a publicly available single-cell RNA-seq dataset accessible from https://singlecell.broadinstitute.org/single_cell/study/ SCP867/hca-lungmap-covid-19-barbry-lung?scpbr=hca-covid-19-int egrated-analysis ${ }^{31}$. Gene-expression analysis on human data shown in Supplementary Table 2 (GSEA_Mason's paper) refers to the RNA-seq dataset in ref. ${ }^{30}$.

53. Chan, J. F. et al. A familial cluster of pneumonia associated with the 2019 novel coronavirus indicating person-to-person transmission: a study of a family cluster. Lancet 395, 514-523 (2020).

54. Chu, H. et al. Comparative tropism, replication kinetics, and cell damage profiling of SARS-CoV-2 and SARS-CoV with implications for clinical manifestations, transmissibility, and laboratory studies of COVID-19: an observational study. Lancet Microbe 1, e14-e23 (2020).

55. Yuan, S. et al. SREBP-dependent lipidomic reprogramming as a broad-spectrum antiviral target. Nat. Commun. 10, 120 (2019).

56. Zhou, Y. et al. Metascape provides a biologist-oriented resource for the analysis of systems-level datasets. Nat. Commun. 10, 1523 (2019).

57. Dobin, A. et al. STAR: ultrafast universal RNA-seq aligner. Bioinformatics 29, 15-21 (2013). 
58. Love, M. I., Huber, W. \& Anders, S. Moderated estimation of fold change and dispersion for RNA-seq data with DESeq2. Genome Biol. 15, 550 (2014).

59. Liberzon, A. et al. Molecular signatures database (MSigDB) 3.O. Bioinformatics 27, 1739-1740 (2011).

60. Whitt, M. A. Generation of VSV pseudotypes using recombinant $\triangle G$-VSV for studies on virus entry, identification of entry inhibitors, and immune responses to vaccines. J. Virol. Methods 169, 365-374 (2010).

61. Anson, B. J. et al. Broad-spectrum inhibition of coronavirus main and papain-like proteases by HCV drugs. Preprint at https://doi.org/10.21203/rs.3.rs-26344/v1 (2020).

62. Ratia, K. et al. A noncovalent class of papain-like protease/deubiquitinase inhibitors blocks SARS virus replication. Proc. Natl Acad. Sci. USA 105, 16119-16124 (2008).

63. Báez-Santos, Y. M. et al. X-ray structural and biological evaluation of a series of potent and highly selective inhibitors of human coronavirus papain-like proteases. J. Med. Chem. 57, 2393-2412 (2014).

64. Ghosh, A. K. et al. Design, synthesis and antiviral efficacy of a series of potent chloropyridyl ester-derived SARS-CoV 3CLpro inhibitors. Bioorg. Med. Chem. Lett. 18, 5684-5688 (2008).

65. Bliss, C. I. The toxicity of poisons applied jointly. Ann. Appl. Biol. 26, 585-615 (1939).

66. Jacob, A. et al. Derivation of self-renewing lung alveolar epithelial type II cells from human pluripotent stem cells. Nat. Protoc. 14, 3303-3332 (2019).

67. Ghaedi, M. et al. Human iPS cell-derived alveolar epithelium repopulates lung extracellular matrix. J. Clin. Invest. 123, 4950-4962 (2013).

68. Chu, H. et al. Comparative replication and immune activation profiles of SARS-CoV-2 and SARS-CoV in human lungs: an ex vivo study with implications for the pathogenesis of COVID-19. Clin. Infect. Dis. ciaa410 (2020).

Acknowledgements The following reagent was deposited by the Centers for Disease Control and Prevention and obtained through BEI Resources, NIAID, NIH: SARS-Related Coronavirus 2, Isolate USA-WA1/2020, NR-52281. The authors thank A. Embry for guidance in securing reagents; S. Yoh for scientific input; $H$. Nymark-McMahon for manuscript editing: T. Marathe for copy editing; S. Blondelle and L. Adelman for facilities and biosafety support; M. Chacon for administrative assistance; W. Thienphrapa and H. Curry for literature research support; R. Eaden for shipping and delivery assistance; O. Harismendy for advice on data analysis; and B. Anson and A. Hamdani in the Mesecar laboratory for providing samples of SARS-CoV-2 Mpro. This work was supported by the following grants to the Sanford Burnham Prebys Medical Discovery Institute: DoD: W81XWH-20-1-0270; DHIPC: U19 Al118610; Fluomics/NOSI: U19 Al135972, as well as generous philanthropic donations from D. Ruch and S. Blair and J. Blair. The work at the University of Hong Kong was partly supported by the donations of R. Yu and C. Yu, the Shaw Foundation of Hong Kong,

M. S.-K. Tong and M. T. M. M. Yin; and funding from the Health and Medical Research Fund (grant no. COVID190121), the Food and Health Bureau, The Government of the Hong Kong Special Administrative Region; the National Program on Key Research Project of China (grant no. 2020YFA0707500 and 2020YFA0707504); and the Theme-Based Research Scheme (T11/707/15) of the Research Grants Council and Hong Kong Special Administrative Region. Viral protease studies have been funded in part with federal funds from the National
Institute of Allergy and Infectious Diseases, National Institutes of Health, Department of Health and Human Services, under Contract No. HHSN272201700060C to A.D.M. M.E.C. is supported by an NIH/NIGMS T32 Training Grant for Structural Biology and Biophysics (GM132024). The work on iPCS-derived pneumocytes was partly supported by the Huffington Foundation. The work at Calibr at Scripps Research was supported by the Bill and Melinda Gates Foundation. This research was also partly funded by CRIP (Center for Research for Influenza Pathogenesis), a NIAID supported Center of Excellence for Influenza Research and Surveillance (CEIRS, contract no. HHSN272201400008C), by DARPA grant HR0011-19-2-0020, by an administrative supplement to NIAID grant U19AI142733, and by the generous support of the JPB Foundation, the Open Philanthropy Project (research grant 2020-215611 (5384)) and anonymous donors to A.G.-S. and by supplements to NIAID grant U19A1135972 and DoD grant W81XWH-20-1-0270 to S.K.C. and A.G.-S. The funding sources had no role in the study design, data collection, analysis, interpretation or writing of the report. Finally, we wish to thank the late Ananda Mohan Chakrabarty for his generous and inspiring mentorship and advice.

Author contributions L.R., S.Y., X.Y., P.T., T.-T.H.N., J.F.-W.C., P.D.D.J., M.V.H., J.C., V.K.-M.P., A.R., Y.P., C.N., A.C., R.R., M.S., M.D., M.E.C. and E.K.L. designed and/or performed experiments. L.R., S.Y., X.Y., N.M., S.B.-M., L.P., K.M.H., M.W.C., K.C., M.S., M.E.C., E.K.L., A.D.M., C.B., A.I.S., R.J.G. and P.T. analysed data. L.R., S.Y., X.Y., L. Martin-Sancho, L.M., M.D., T.P.Z., L. Martinez-Sobrido, W.-C.L., K.M.W., R.A., J.R.J. and K.-Y.S. generated critical reagents. E.R., R.S., P.G.S., A.D.M., A.G.-S., A.K.C., K.-Y.Y. and S.K.C. oversaw the conception and design of the experiments. L.R., X.Y., N.M., L. Martin-Sancho, K.M.H. and S.K.C. wrote the manuscript.

Competing interests J.F.-W.C. has received travel grants from Pfizer Corporation Hong Kong and Astellas Pharma Hong Kong Corporation Limited and was an invited speaker for Gilead Sciences Hong Kong Limited and Luminex Corporation. A.G.-S. is inventor on patent applications on antiviral compounds against SARS-CoV-2 unrelated to this study and owned by the Icahn School of Medicine at Mount Sinai. A.K.C. and S.K.C. are inventors on a patent application on repurposed antiviral compounds for SARS-CoV-2 owned by Scripps Research and Sanford Burnham Prebys. US Patent Application Serial No. 63/010630, entitled Methods and Compositions for Antiviral Treatment relates to aspects of this work and was filed on 15 April 2020. The corresponding authors had full access to all the data in the study and had final responsibility for the decision to submit for publication. The other authors declare no competing interests.

Additional information

Supplementary information is available for this paper at https://doi.org/10.1038/s41586-020 2577-1.

Correspondence and requests for materials should be addressed to A.K.C., K.-Y.Y. or S.K.C. Peer review information Nature thanks Timothy Sheahan, Brian Shoichet and the other, anonymous, reviewer(s) for their contribution to the peer review of this work. Peer reviewer reports are available.

Reprints and permissions information is available at http://www.nature.com/reprints. 
a

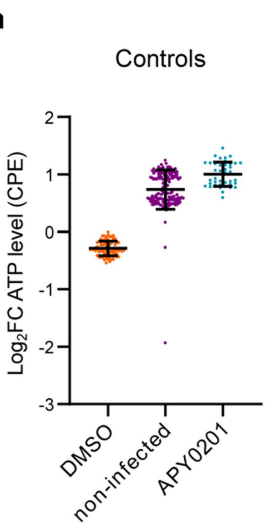

d
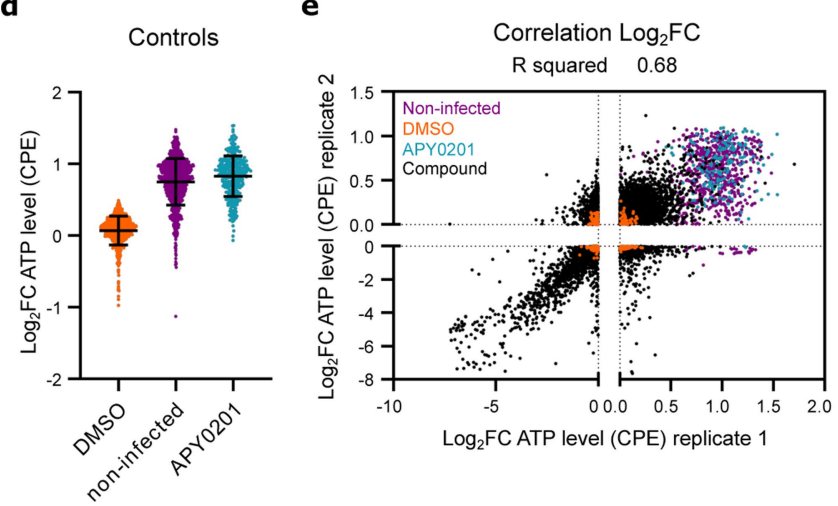
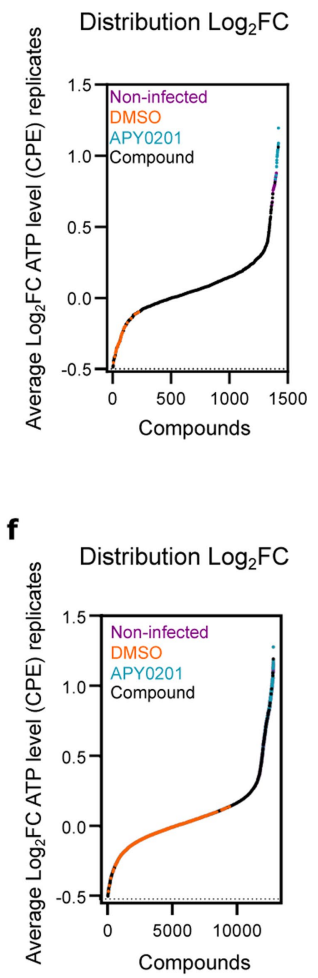

Extended Data Fig. 1 | High-throughput repositioning screens for SARS-CoV-2 antivirals. a-c, Data from preliminary LOPAC1280 library primary screen. d-f, Data from ReFRAME collection screen. a, d, $\log _{2}$ fold change $(\log 2 \mathrm{FC})$ of ATP levels after normalization to the median of each plate for SARS-CoV-2 infected all positive (APY0201) and negative (DMSO) controls, as well as for non-infected cells, across all screening plates. Error bars represent mean \pm s.d. for at least $n=40$ (a) and $n=376$ (d) independent wells. b,e, Correlation plot indicating the $\log _{2} \mathrm{FC}$ of each compound in the two replicate screens. c, f, Distribution of activities for each compound according to the average of the $\log _{2} \mathrm{FC}$ of each replicate. Each datapoint indicates the average $\log _{2} \mathrm{FC}$ of each drug between the screening replicates (black dots). Values corresponding to DMSO (orange dots), APY0201 (cyan dots) and non-infected cells (purple dots) are also represented. Red circles indicate the activities of selected compounds chosen for follow-up for the LOPAC1280 screens. R squared value indicates the linear correlation coefficient for the replicates of LOPAC1280 (b) and ReFRAME (e) screens. 

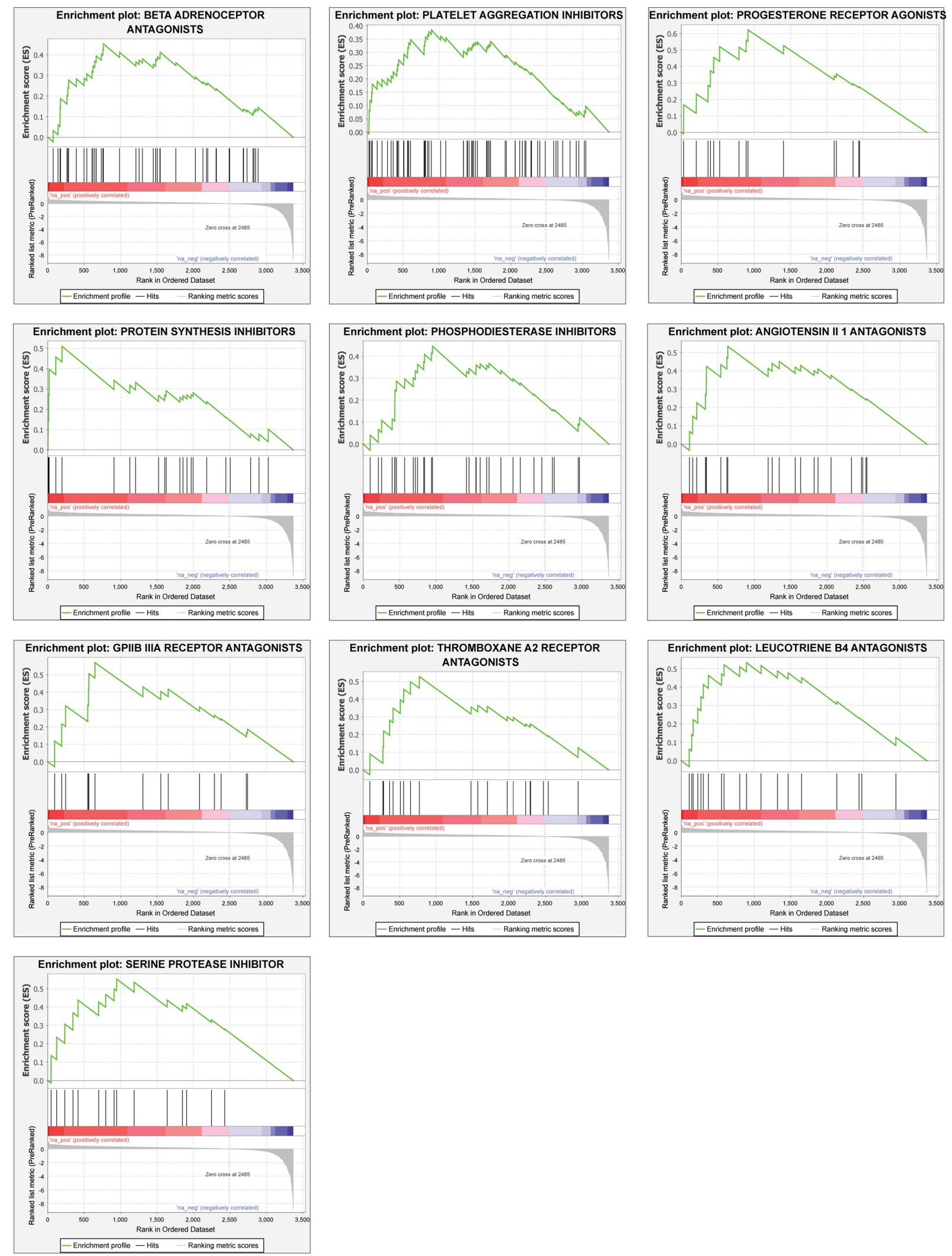

Extended Data Fig. 2 | Supplementary GSEA analysis. Gene set enrichment analysis (GSEA) of primary screening data according to the average $Z$-score. GSEA enrichment plots of additional ten target classes that were enriched in the primary HTS assay are shown, including beta adrenoreceptor antagonists, platelet aggregation inhibitors, progesterone receptor agonists, protein synthesis inhibitors, phosphodiesterase inhibitors, angiotensin II 1 antagonists, GPIIB IIIA receptor antagonists, thromboxane $\mathrm{A} 2$ receptor antagonists, leucotriene $B 4$ antagonists, serine protease inhibitors $(P<0.05$, FDR $q<0.25$ ). $Z$-scores distributions of compound activities within the screen are depicted below each plot (Ranked list metric). $P$ values were calculated as indicated in the Methods. 
a

\section{PPAR signaling pathway}

Regulation of trans-synaptic signaling

Signaling by Nuclear Receptors

Calcium signaling pathway

DNA-template transcription, initiation

Retinoic acid receptor signaling pathway

Transcription initiation of RNA polymerase II promoter

Class A/1 (Rhodopsin-like receptors)

$\mathrm{G}$ protein-coupled receptor signaling pathway

GPCR ligand binding

Nuclear receptor transcription pathway

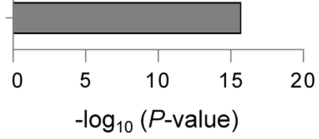

Extended Data Fig. 3 | Enriched biological pathways and processes of putative antiviral compound targets. a, Bar graph of enriched biological pathways and putative proteins targeted by the antiviral compounds identified by HTS analysis. Molecular targets contained within enriched GSEA classes, as well as those of the 326 compounds selected for validation, were assessed for enrichment of pathways and biological functions. The $x$ axis corresponds to $-\log _{10}(P$ value $)$ while the $y$ axis indicates the enriched terms. The analysis was performed using the online tool Metascape and $P$ values were calculated as indicated in the Methods. b, Chemical epistasis analysis of GPCR agonists and antagonists on viral replication. Vero E6 cells were treated with antagonists of the serotonin receptor $1 \mathrm{~A}$ (NAD 299 hydrochloride, $5 \mu \mathrm{M}$ ), serotonin receptor b
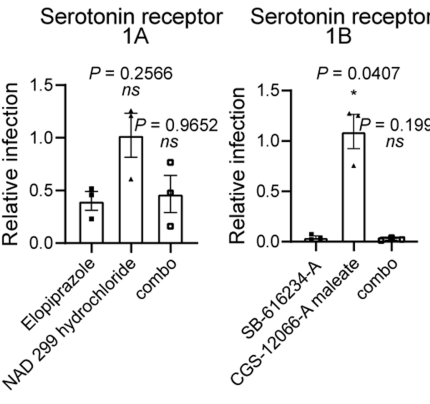

Dopamine $D 2$
and $D 3$ receptors

Platelet-Activating and D3 receptors Factor Receptor $P=0.0991$ $P=0.0929$
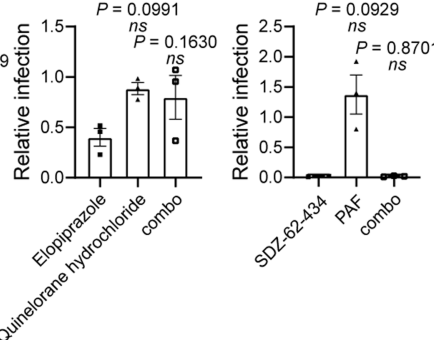

Serotonin receptor $1 \mathrm{~A}$ $P=0.9652$
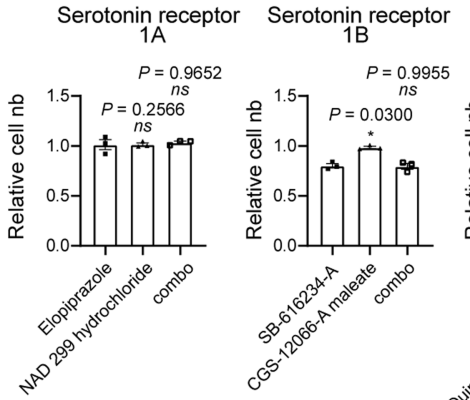

Dopamine D2 and D3 receptors $P=\underset{n s}{0.9964}$
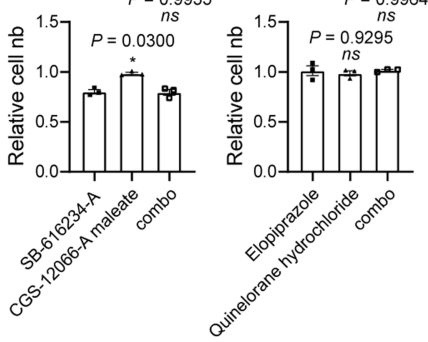

Platelet-Activating Factor Receptor

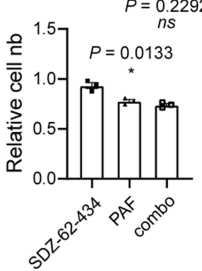

1B (SB-616234-A, $2.5 \mu \mathrm{M}$ ), Dopamine D2 and D3 receptors (elopiprazole, $5 \mu \mathrm{M}$ ) and Platelet-Activating Factor (PAF) receptor (SDZ-62-434, 5 $\mu \mathrm{M}$ ) and challenged with SARS-CoV-2. Infection was determined in the top panels as described in Fig. 3. Similarly, Vero E6 cells where pretreated with an agonist of the serotonin receptor $1 \mathrm{~A}$ (elopiprazole, $5 \mu \mathrm{M}$ ), serotonin receptor $1 \mathrm{~B}$ (CGS12066-A maleate, $2.5 \mu \mathrm{M}$ ), Dopamine D2 and D3 receptors (quinelorane hydrochloride, $5 \mu \mathrm{M}$ ) and Platelet-Activating Factor (PAF) receptor (PAF, $5 \mu \mathrm{M})$, either alone or in combination (combo) with the corresponding antagonist. Cellular toxicity was measured through enumeration of cell numbers (bottom panels). Data are normalized to the average of DMSO-treated wells and represent mean \pm s.e.m. for $n=3$ independent experiments. 


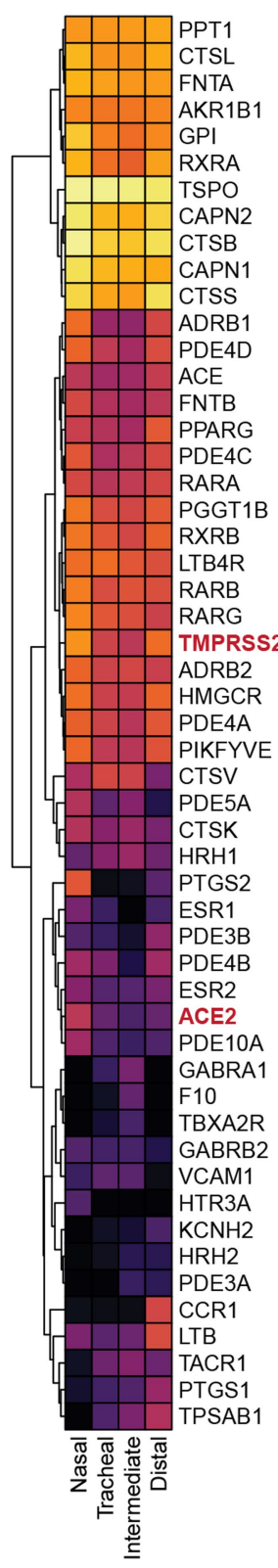

Location

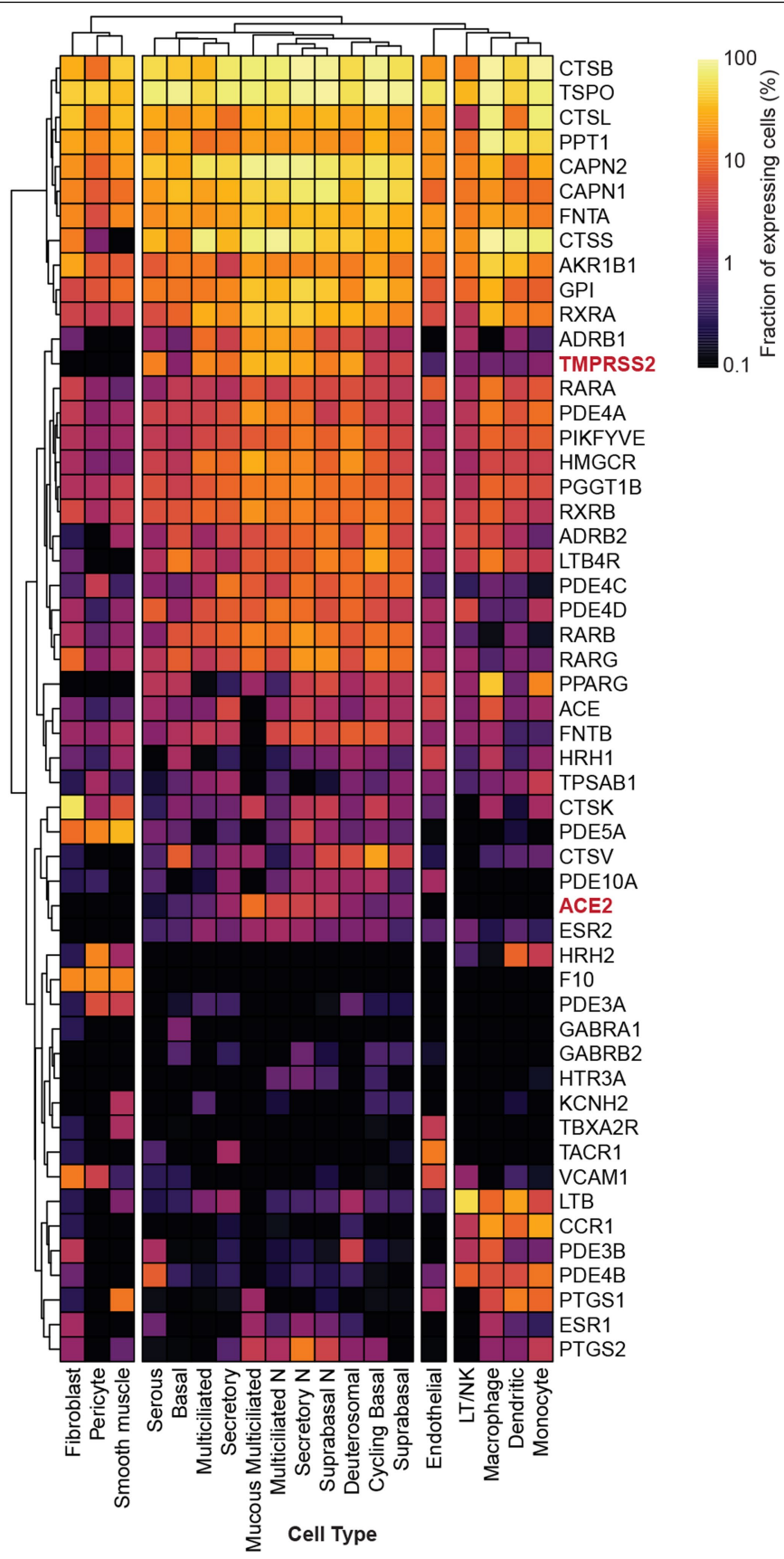

Extended Data Fig. 4 | Expression profiles of compound-targeted genes in human airway samples. Expression profiles of ACE2, TMPRSS2, and indicated targets of putative antiviral compounds identified in the HTS screen was analysed using previously reported single-cell RNA profiling data from human airway samples of healthy donors. Clustered heat maps show the fraction of gene-expressing cells separated by sampling locations (left panel) or cell type (right panel). 


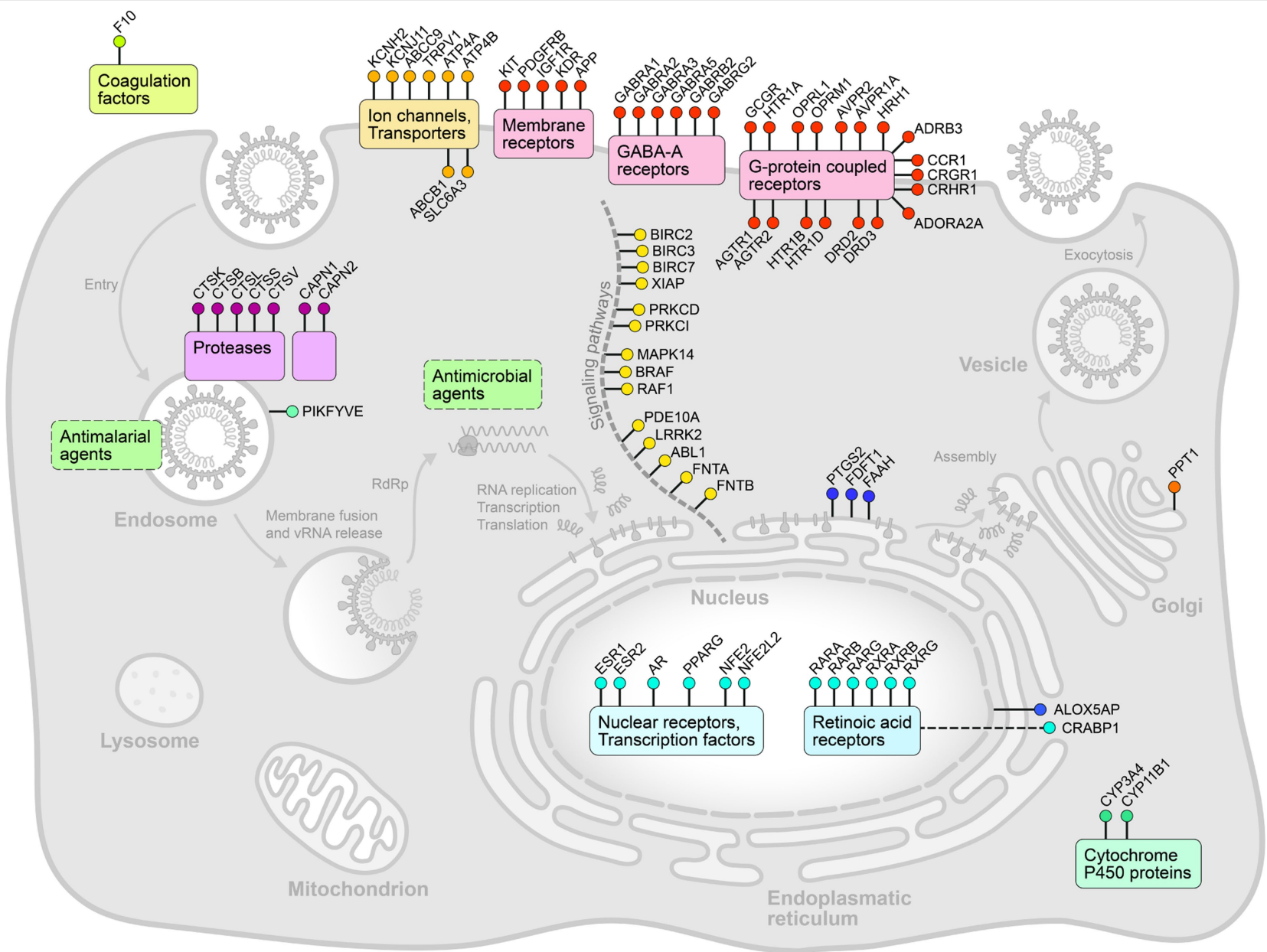

Extended Data Fig. 5 | A cellular map of SARS-CoV-2 antiviral targets. Reported targets or target classes of confirmed SARS-CoV-2 antiviral compounds (Supplementary Table1) were mapped to a cell based on known or inferred subcellular localization, function, and potential intersection with the viral life cycle. 
Phosphoinositide kinase
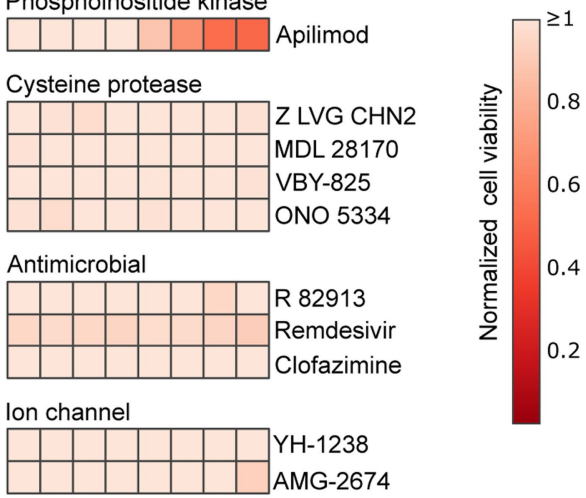

$\mathrm{G}$ protein-coupled receptor

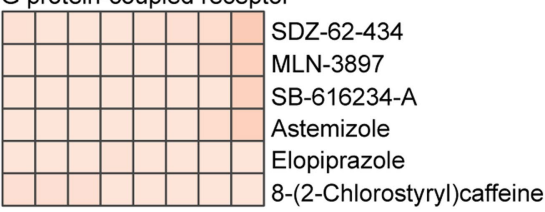

Chloroquine derivative

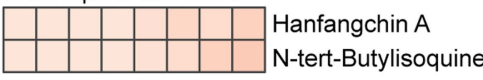

Miscellaneous

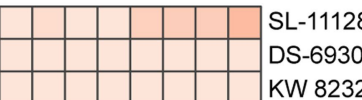

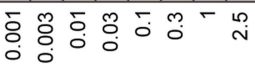

Concentration $[\mu \mathrm{M}]$
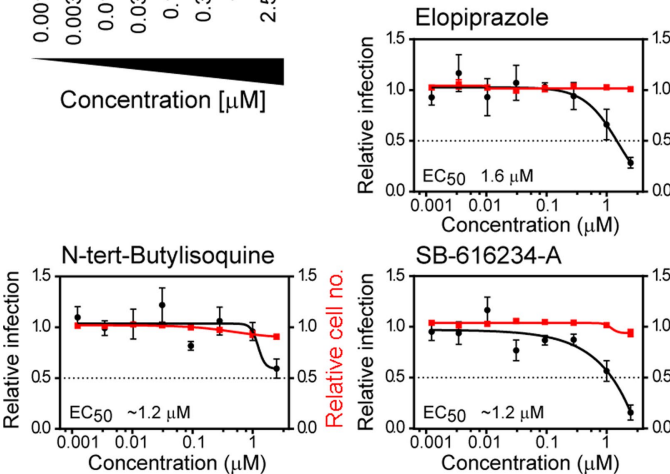

Concentration $(\mu \mathrm{M})$

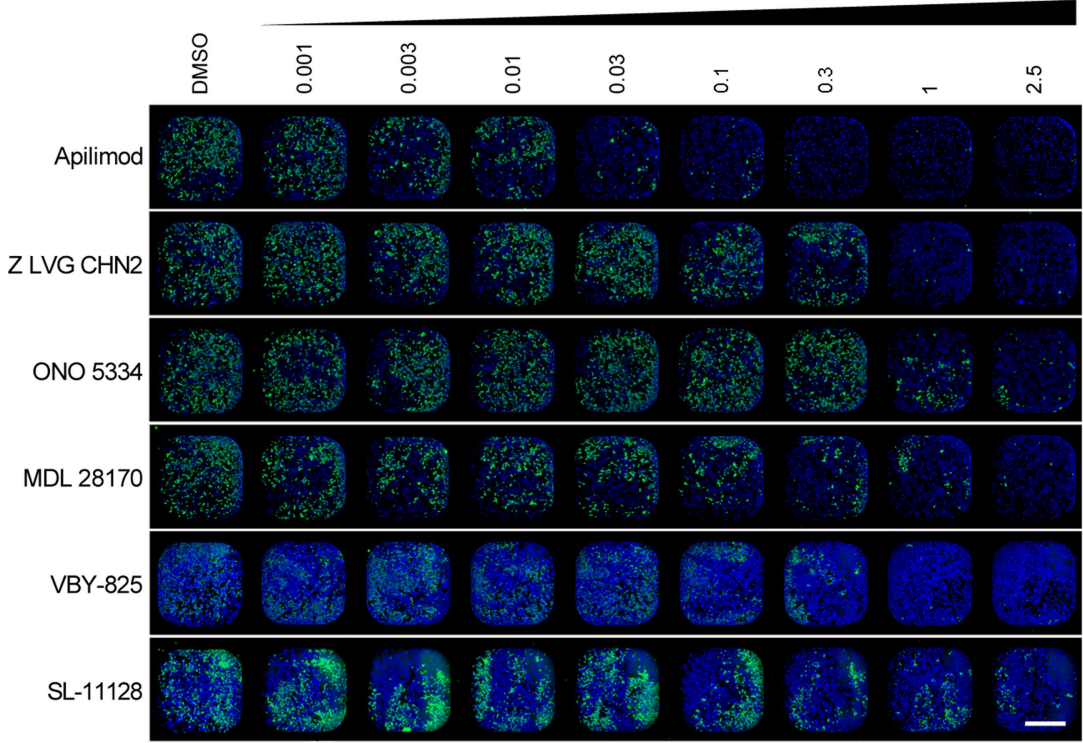

C
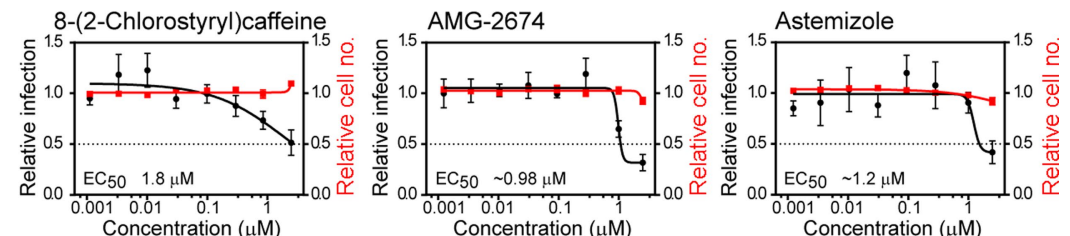

Hanfangchin A

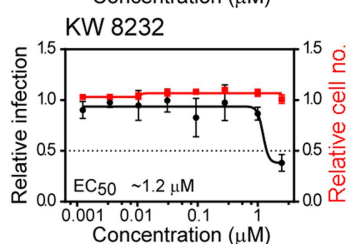

MLN-3897

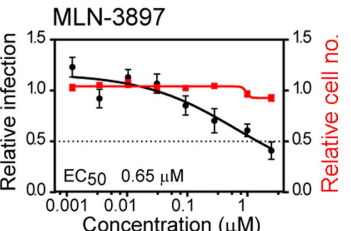

$\mathrm{YH}-1238$
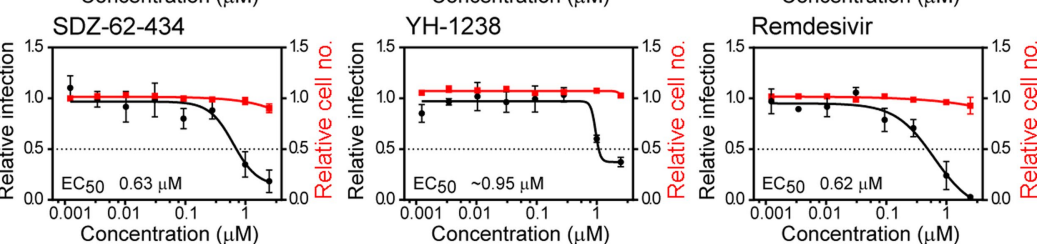

Concentration $(\mu \mathrm{M})$

Extended Data Fig. 6 | Cell number and IF relative to dose-response orthogonal validation in Vero E6 cells. a, Vero E6 cells were pre-treated for $16 \mathrm{~h}$ with increasing concentrations of the indicated compound and then infected with SARS-CoV-2 with $\mathrm{MOI}=0.1 .24 \mathrm{~h}$ post-infection, cells were fixed, and immunostained, and imaged. For each condition, the total amount of cells stained with DAPI was calculated. Data are normalized to the average of DMSO-treated wells. The heat map represents the normalized cell number of the indicated 21 compounds in dose-response, on a scale from 0 to 1 , on the average of five independent experiments. Compounds are grouped in predicted functional clusters. Concentrations are rounded. Corresponding antiviral activities of these compounds are shown in Fig. 3a.b, Representative immunofluorescence images corresponding to one of the three dose- responses illustrated in Fig. 3. For each condition, the corresponding entire well is shown ( $4 \mathrm{x}$ objective). Scale bar $=1.35 \mathrm{~mm}$. c, Dose-response curves for additional antiviral compounds. Vero $\mathrm{E} 6$ cells were pre-treated for $16 \mathrm{~h}$ with increasing concentrations of the indicated compound and then infected with SARS-CoV-2 with MOI $=0.1$ in the presence of the compound. $24 \mathrm{~h}$ post-infection, cells were fixed, and an immunofluorescence was performed. For each condition, the percentage of infection was calculated as the ratio between the number of infected cells stained for CoV NP and the total amount of cells stained with DAPI. Dose-response curves for both infectivity (black) and cell number (red) are shown. Data are normalized to the average of DMSO-treated wells and represent mean \pm s.e.m. for $n=5$ independent experiments. 
a 293T-ACE2 cells
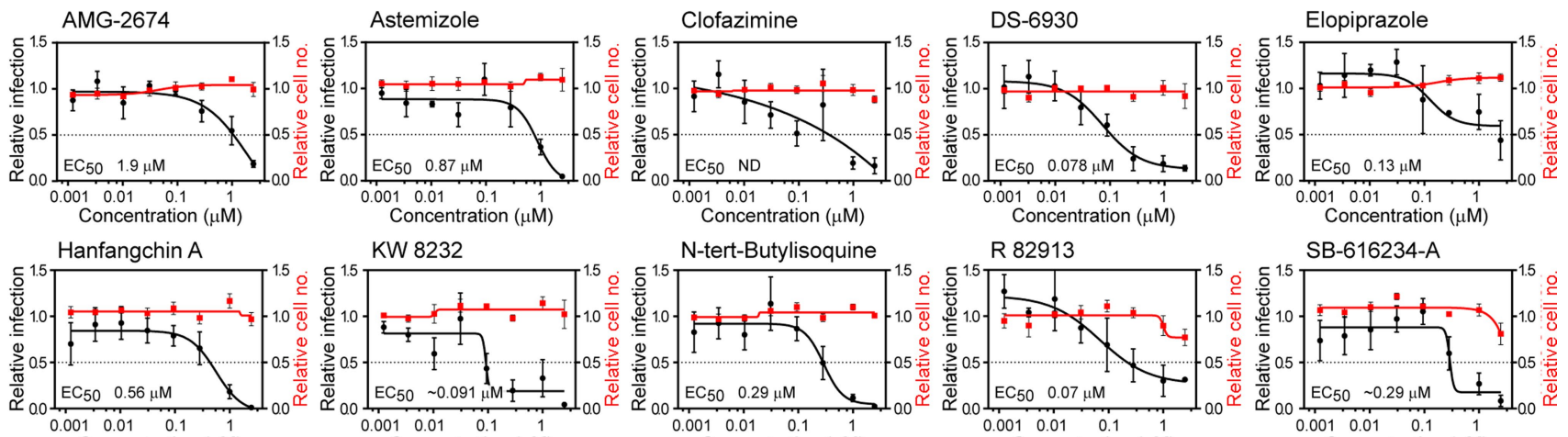

KW 8232

Concentration $(\mu \mathrm{M})$

R 82913

Concentration $(\mu \mathrm{M})$

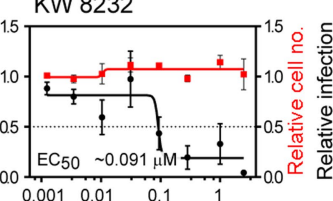

$\mathrm{N}$-tert-Butylisoquine
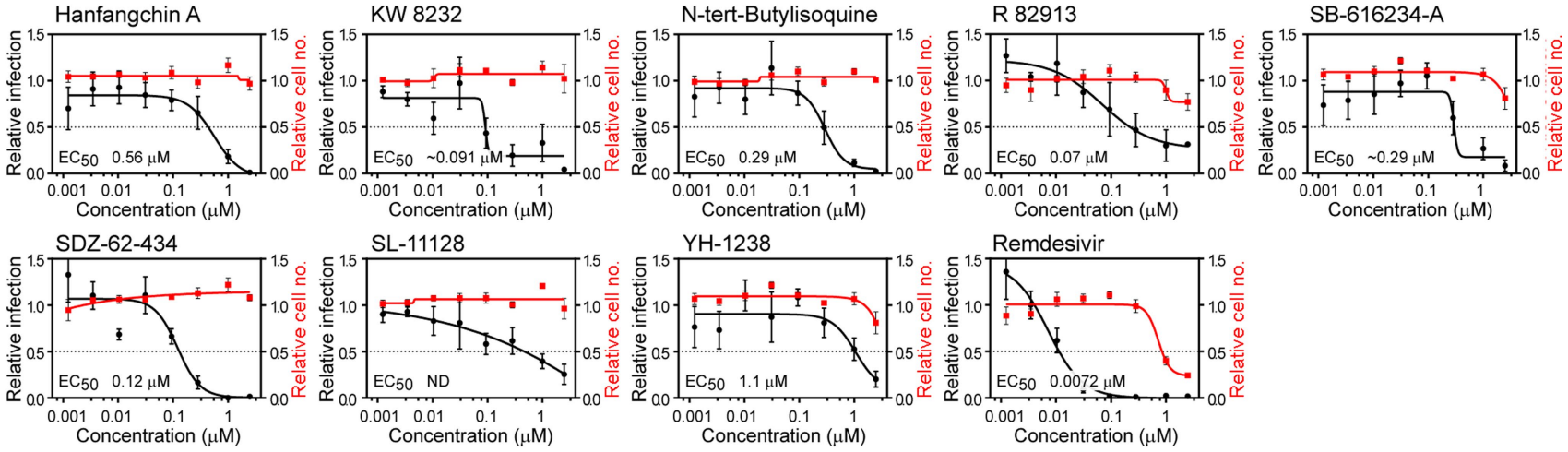

b

Huh-7-ACE2 cells
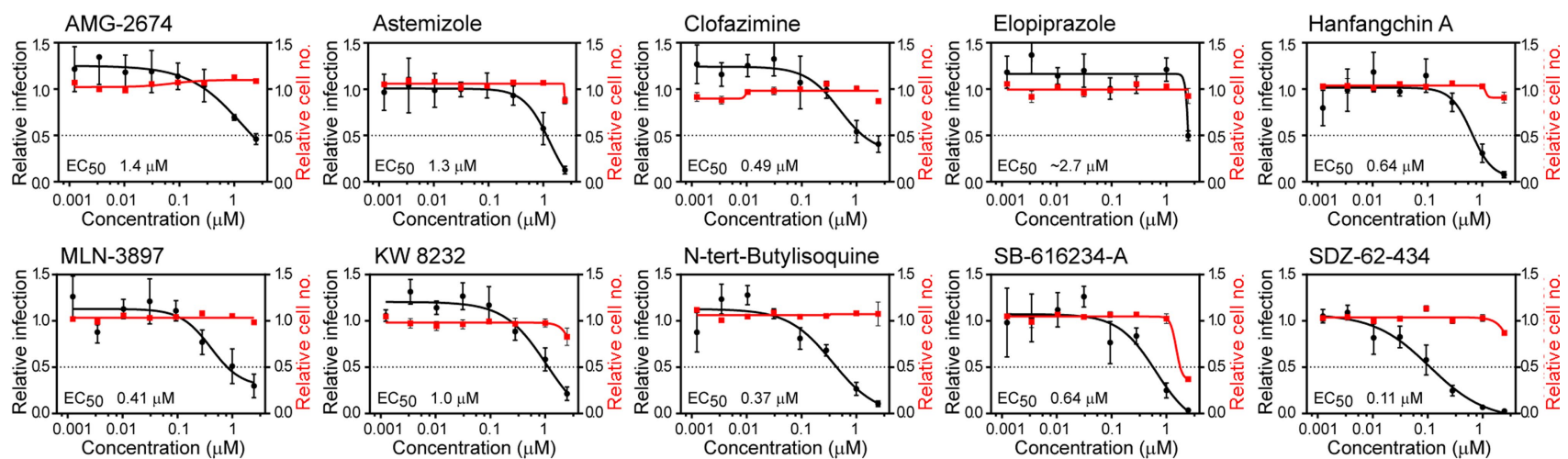
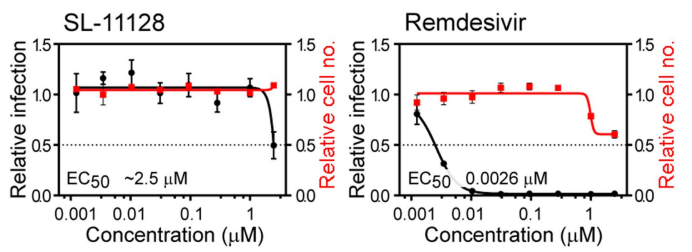

Extended Data Fig. 7 | Dose-response curves of additional antiviral compounds in HEK293T-ACE2 and Huh-7-ACE2 expressing cells. HEK-293T (a) and Huh-7 cells (b), both transduced with ACE2, were pre-treated for $16 \mathrm{~h}$ with increasing concentrations of the indicated compound and then infected with SARS-CoV-2 with $\mathrm{MOI}=0.3$ (a) or $0.2(\mathbf{b})$, in the presence of the compound. $24 \mathrm{~h}$ post-infection, cells were fixed, and immunostained, followed by imaging. For each condition, the percentage of infection was calculated as the ratio between the number of infected cells stained for CoV NP and the total amount of cells stained with DAPI. Compound concentrations range between $1 \mathrm{nM}$ and $2.5 \mu \mathrm{M}$ with three-fold dilutions. Dose-response curves for both infectivity (black) and cell number (red) are shown. Data are normalized to the average of DMSO-treated wells and represent mean \pm s.e.m. for $n=4$ independent experiments. $\mathrm{EC}_{50}$ values for each compound were calculated as 4-parameter logistic nonlinear regression model and are indicated. 
a

SARS-CoV-2 3CLpro

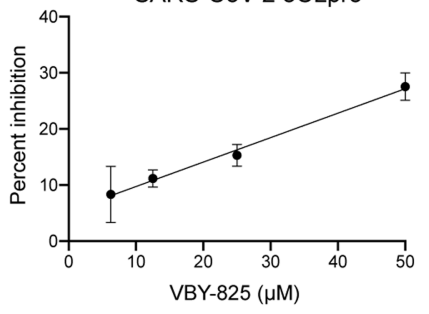

b

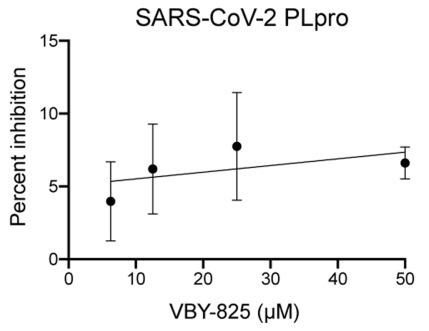

SARS-CoV-2 3CLpro

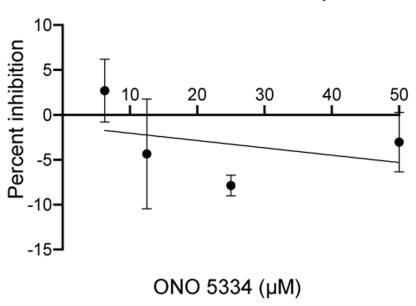

ONO $5334(\mu \mathrm{M})$

SARS-CoV-2 PLpro

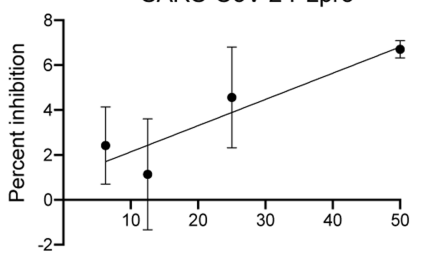

ONO $5334(\mu \mathrm{M})$

Extended Data Fig. 8 | In vito protease assay on SARS-CoV-2 $\mathbf{P L}_{\text {pro }}$ and main protease $\mathbf{M}_{\text {pro }} \cdot \mathbf{a}$, b, Purified SARS-CoV-2 Moro (a) and SARS-CoV-2 PLpro (b) enzymes were incubated with varying concentrations of each compound, ranging from 1 to $50 \mu \mathrm{M}$. Activity of purified SARS-CoV- $2 \mathrm{M}_{\text {pro }}$ and SARS-CoV-2 $\mathrm{PL}_{\text {pro }}$ enzymes was measured using the UIVT-3 peptide substrate (HiLyte

SARS-CoV-2 3CLpro

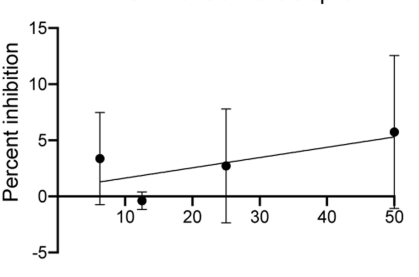

Z LV CHN2 ( $\mu \mathrm{M})$

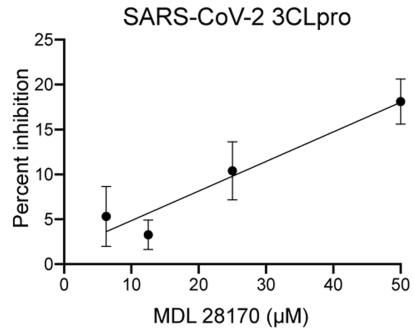

SARS-CoV-2 PLpro

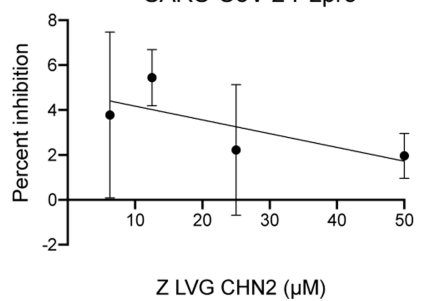

Z LV CHN2 $(\mu \mathrm{M})$
SARS-CoV-2 PLpro

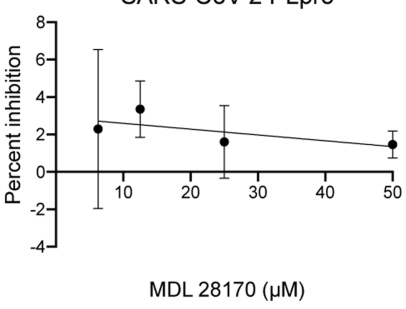

Fluor488-ESATLQSGLRKAK-QXL520-NH2) and the peptide substrate Arg-Leu-Arg-Gly-Gly-AMC (RLRGG-AMC) respectively. Enzyme activity in the absence (zero per cent inhibition control) and presence of compounds were used to calculate the per cent inhibition at each compound concentration. Data are presented as mean \pm sod. for $n=3$ independent experiments. 


\section{Article}

a

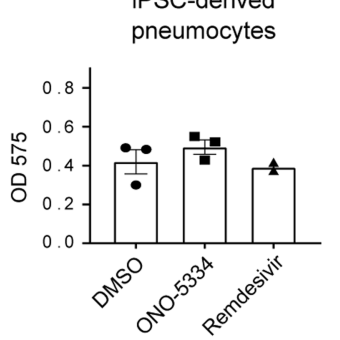

b

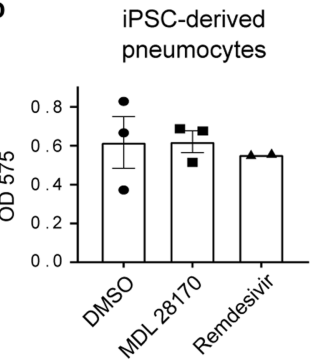

c

iPSC-derived

pneumocytes

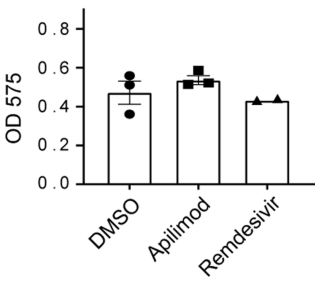

Extended Data Fig. 9 |Cell viability in human iPSC-derived pneumocyte-like cells. a-c, MTT assay performed on human iPSC-derived pneumocyte-like cells

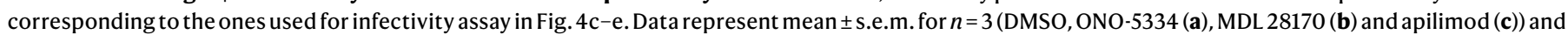
$n=2$ (remdesivir) biological replicates. 

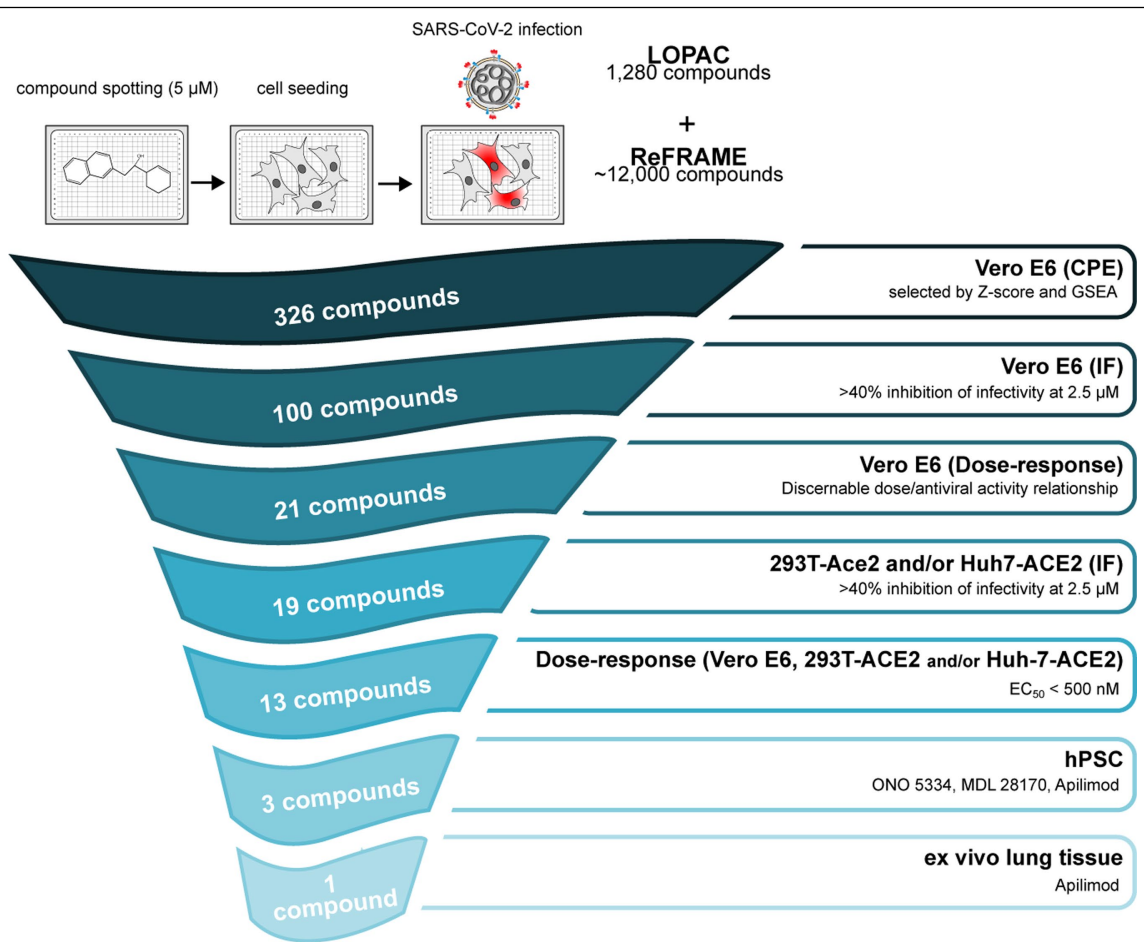

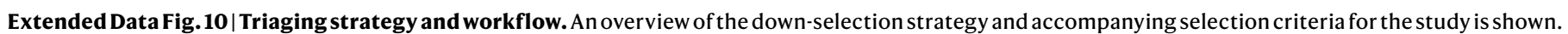




\section{natureresearch}

Corresponding author(s): Sumit K. Chanda

Last updated by author(s): Jul 5, 2020

\section{Reporting Summary}

Nature Research wishes to improve the reproducibility of the work that we publish. This form provides structure for consistency and transparency in reporting. For further information on Nature Research policies, seeAuthors \& Referees and theEditorial Policy Checklist .

\section{Statistics}

For all statistical analyses, confirm that the following items are present in the figure legend, table legend, main text, or Methods section.

n/a Confirmed

$\boldsymbol{x}$ The exact sample size $(n)$ for each experimental group/condition, given as a discrete number and unit of measurement

x A statement on whether measurements were taken from distinct samples or whether the same sample was measured repeatedly

The statistical test(s) used AND whether they are one- or two-sided

Only common tests should be described solely by name; describe more complex techniques in the Methods section.

$\mathbf{x} \square$ A description of all covariates tested

$\mathbf{x} \square$ A description of any assumptions or corrections, such as tests of normality and adjustment for multiple comparisons

x A full description of the statistical parameters including central tendency (e.g. means) or other basic estimates (e.g. regression coefficient)

$\boldsymbol{x}$ AND variation (e.g. standard deviation) or associated estimates of uncertainty (e.g. confidence intervals)

For null hypothesis testing, the test statistic (e.g. $F, t, r$ ) with confidence intervals, effect sizes, degrees of freedom and $P$ value noted

x Give $P$ values as exact values whenever suitable.

$\mathbf{x} \square$ For Bayesian analysis, information on the choice of priors and Markov chain Monte Carlo settings

$\boldsymbol{x} \square$ For hierarchical and complex designs, identification of the appropriate level for tests and full reporting of outcomes

$\boldsymbol{x} \square$ Estimates of effect sizes (e.g. Cohen's $d$, Pearson's $r$ ), indicating how they were calculated

Our web collection on statistics for biologists contains articles on many of the points above.

\section{Software and code}

Policy information about availability of computer code
Data collection
No software was used

Data analysis

The following softwares and websites were used for the analyses in this study: STAR 2.7.3a, R 3.6.3, DESeq2 1.26.0, fgsea 1.12.0, GSEA

4.0.3, Graph Pad Prism 8.4.1, CortellisTM (Clarivate Analytics) and drugs.com websites (analyses ran between April and June 2020),

Metascape website (analysis ran in April 2020)

For manuscripts utilizing custom algorithms or software that are central to the research but not yet described in published literature, software must be made available to editors/reviewers. We strongly encourage code deposition in a community repository (e.g. GitHub). See the Nature Research guidelines for submitting code \& software for further information.

\section{Data}

Policy information about availability of data

All manuscripts must include a data availability statement. This statement should provide the following information, where applicable:

- Accession codes, unique identifiers, or web links for publicly available datasets

- A list of figures that have associated raw data

- A description of any restrictions on data availability

The following data availability statement was added to the manuscript:

Data is available in the supplemental Tables S1, S2, S3 and S4, and through https://reframedb.org (assay A00440). Complete sequences of SARS-CoV-2 HKU-001a and SARS-CoV-2 USA-WA1/2020 are available through GenBank (accession number MT230904, and MT246667 and MN908947 respectively). RNAseq data in supplementary Table S2 were aligned with the genome of the African green monkey (Chlorocebus sabaeus, https://uswest.ensembl.org/Chlorocebus_sabaeus/Info/ Annotation), and with the SARS-CoV-2 genome (https://www.ncbi.nlm.nih.gov/nuccore/NC 045512), selected as the reference genome. The dataset is available on GEO with accession number GSE153940. Figure ED3 derived from the analysis of publicly available single-cell RNA-seq dataset accessible at https:// singlecell.broadinstitute.org/single cell/study/SCP867/hca-lungmap-covid-19-barbry-lung?scpbr=hca-covid-19-integrated-analysis44. Gene expression analysis on human data shown in supplementary Table S2 (GSEA_Mason's paper) refers to the RNA-seq dataset available at https://www.biorxiv.org/ 


\section{Field-specific reporting}

Please select the one below that is the best fit for your research. If you are not sure, read the appropriate sections before making your selection.
$\boldsymbol{x}$ Life sciences
$\square$ Behavioural \& social sciences
Ecological, evolutionary \& environmental sciences

For a reference copy of the document with all sections, see nature.com/documents/nr-reporting-summary-flat.pdf

\section{Life sciences study design}

All studies must disclose on these points even when the disclosure is negative.

Sample size Vero E6 are a cell line. A Pearson's R-squared coefficient was calculated to assess the sample sizing was acceptable. The R-squared correlation coefficient $>0.65$, obtained from both the LOPAC and ReFRAME primary screens performed in duplicate, supports a good correlation for $n=2$. For dose-responses, Vero E6, HEK-293T and Huh-7 cell lines were used and $n=$ at least 3 independent experiments were performed. In order to increase the statistical reliability for calculating EC50s, for some of the cell lines up to $n=5$ independent experiments were performed (specified in the figure legends).

For hPSC-derived pneumocytes and ex vivo tissue lung samples, experiments were performed with $\mathrm{n}=3$ (except for MTT assay in hPSC treated with the control remdesivir, where $n=2$ was used). The sample size for each experiment is specified in each corresponding figure legend.

Data exclusions No data has been excluded from the analyses presented in this manuscript.

Replication In order to verify the reproducibility of the experimental findings, the large-scale CPE screen was performed in duplicate and the R-squared correlation coefficient is indicated for both LOPAC and ReFRAME screens. The validation screens and all the confirmation studies in human cell models were performed at least in duplicate and the means +/- SEM or SD as well as the nature of ' $n$ ' are indicated in the figure legends. All the attempts were successful and no data was excluded from the analyses.

Randomization The position of each compounds into the plates was randomly chosen for both the primary screen and the further validations.

Blinding Data collection for the primary screen and the validation in Vero E6, 293T-ACE2 and Huh-7-ACE2 was run blinded, without knowing which compound was spot in the specific wells. Data analysis was also blinded, with the investigators only having internal IDs of compounds, without knowing their identity. For small-scale experiments (time-of-addition, VLPs, iPSC-derived cells, ex vivo tissues) the blinding was not relevant since the endpoint was determined independently from the final readout.

\section{Reporting for specific materials, systems and methods}

We require information from authors about some types of materials, experimental systems and methods used in many studies. Here, indicate whether each material, system or method listed is relevant to your study. If you are not sure if a list item applies to your research, read the appropriate section before selecting a response.

\section{Materials \& experimental systems}

\begin{tabular}{l|l|l}
\hline n/a & Involved in the study \\
\hline & $\boldsymbol{x}$ & Antibodies \\
\hline$\square$ & $\boldsymbol{x}$ & Eukaryotic cell lines \\
\hline $\boldsymbol{x}$ & $\square$ & Palaeontology \\
\hline $\boldsymbol{x}$ & $\square$ Animals and other organisms \\
\hline & $\boldsymbol{x}$ & Human research participants \\
\hline $\boldsymbol{x}$ & $\square$ Clinical data
\end{tabular}

\author{
Methods \\ n/a Involved in the study \\ $\boldsymbol{x} \square$ ChIP-seq \\ $\square \quad x$ Flow cytometry \\ $\mathbf{x} \square$ MRI-based neuroimaging
}

\section{Antibodies}

\section{Antibodies used}

Validation
Rabbit-anti-SARS-CoV-1 nucleoprotein serum, which exhibits strong cross-reactivity with SARS-CoV-2 (A-G Sastre, unpublished data)(1:10,000 dilution). Alexa Fluor 488-conjugated goat-anti-rabbit IgG (Thermo Fisher Scientific, USA; Catalog \# A27034) (1:2,000 dilution).

The antibody was tested for cross-reactivity with SARS-CoV2 in Vero E6 cells. The antibody showed specificity to SARS-CoV-2infected cells and no background in non-infected cells. 
Policy information about cell lines

Cell line source(s)

Vero E6 and HEK-293T cells were obtained from ATCC (ATCC ${ }^{\circledR}$ CRL-1586 and ATCC ${ }^{\oplus}$ CRL-3216 TM respectively). Huh-7 were obtained from Apath LLC and BHK-21/WI-2 cells from Kerafast. hPSC1 cells (H9) were obtained from WiCell. hPSC2 (Lis38derived) were kindly provided by Dr. Jacob Hanna(ISM ESCRO Project \#14-005).

Authentication

All the cell lines were commercially available and have not been authenticated after receiving them.

Mycoplasma contamination

All cells were tested negative for mycoplasma contamination, except for Huh7-Ace2 cells which were tested positive.

Commonly misidentified lines

(See ICLAC register)

No commonly misidentified cell lines were used in this study.

\section{Human research participants}

Policy information about studies involving human research participants

Population characteristics This doesn't apply to this study, since tissues from only one donor were used.

Recruitment $\quad$ No patients were recruited for this study. Biopsy samples that would have been otherwise discarded were used for experimental analyses.

Ethics oversight

The donors gave written consent as approved by the Institutional Review Board of the University of Hong Kong/Hospital Authority Hong Kong West Cluster (UW13-364).

Note that full information on the approval of the study protocol must also be provided in the manuscript.

\section{Flow Cytometry}

\section{Plots}

Confirm that:

$\boldsymbol{x}$ The axis labels state the marker and fluorochrome used (e.g. CD4-FITC).

$\boldsymbol{x}$ The axis scales are clearly visible. Include numbers along axes only for bottom left plot of group (a 'group' is an analysis of identical markers).

x All plots are contour plots with outliers or pseudocolor plots.

$\boldsymbol{x}$ A numerical value for number of cells or percentage (with statistics) is provided.

\section{Methodology}

Sample preparation

Cells were detached from the culture plate using $1 \mathrm{ml}$ of enzyme-free dissociation buffer (Sigma) and fixed by adding $1 \mathrm{ml}$ of $10 \%$ formaldehyde for $24 \mathrm{~h}$ at room temperature. Cells were washed once by with Perm/Wash buffer (BD) and stained for the SARS N with in house produced mouse IgG2a monoclonal antibody conjugated to AF647. After $1 \mathrm{~h}$ incubation at room temperature, cells were washed in phosphate buffered saline supplemented with $2 \mathrm{mM}$ EDTA once and resuspended in 200 microliter for analysis.

Instrument

Beckman Coulter Gallios

Software

Kaluza software version 1.0

Cell population abundance

flow cytometry to quantitate virus-infected cells but did not sort them. therefore the question for post sort abundance is actually irrelevant.

Gating strategy

Cells were first gated on singlets by plotting FSC-A versus FSC-H. All single cells were selected in a plot in which FSC-A was plotted versus SSC-A. Within this gate, virus-positive cells were quantified by plotting SSC-A versus SARS-N AF647.

$\mathbf{x}$ Tick this box to confirm that a figure exemplifying the gating strategy is provided in the Supplementary Information. 